\title{
Equivariant Yamabe problem with boundary
}

\author{
Pak Tung Ho $\mathrm{Ho}^{1,2}$. Jinwoo Shin ${ }^{2}$
}

Received: 2 June 2021 / Accepted: 4 December 2021 / Published online: 16 January 2022

(c) The Author(s) 2021

\begin{abstract}
As a generalization of the Yamabe problem, Hebey and Vaugon considered the equivariant Yamabe problem: for a subgroup $G$ of the isometry group, find a $G$-invariant metric whose scalar curvature is constant in a given conformal class. In this paper, we study the equivariant Yamabe problem with boundary.
\end{abstract}

Mathematics Subject Classification Primary 53C18 · 53C21 - Secondary 35R01 · 58J32

\section{Introduction}

Let $M$ be a closed (that is, compact without boundary) $n$-dimensional manifold with $n \geq 3$, and $g$ a Riemannian metric on $M$. As a generalization of the Uniformization Theorem for surfaces, the Yamabe problem [22] is to find a metric conformal to $g$ such that its scalar curvature is constant. This was solved by Trudinger [20], Aubin [2], and Schoen [17].

In 1993, a question was raised by Hebey and Vaugon in [10] as to whether the Yamabe problem could be extended under the control of its isometry group. This problem is called the equivariant Yamabe problem (or Hebey-Vaugon Conjecture). To state it, we need the following definitions. Denote $[g]$ the conformal class of $g$. The group of all conformal transformations of $(M, g), C(M, g)$, is defined as

$$
\left\{f: M \rightarrow M \text { is diffeomorphism } \mid f^{*} g \in[g]\right\} .
$$

The group of all isometries in $(M, g), I(M, g)$, is defined as

$$
\left\{f: M \rightarrow M \text { is diffeomorphism } \mid f^{*} g=g\right\} .
$$

It is clear that $I(M, g) \subseteq C(M, g)$. Then we have the following:

Communicated by Neil S. Trudinger.

Pak Tung Ho

paktungho@yahoo.com.hk

Jinwoo Shin

shinjin@kias.re.kr

1 Department of Mathematics, Sogang University, Seoul 04107, Korea

2 Korea Institute for Advanced Study, Hoegiro 85, Seoul 02455, Korea 
Conjecture 1.1 (Equivariant Yamabe problem) Given a compact subgroup $G$ of $I(M, g)$, there exists a $G$-invariant metric conformal to $g$ such that its scalar curvature is constant.

We say that a metric $\widetilde{g}$ is $G$-invariant if $f^{*} \widetilde{g}=\widetilde{g}$ for all $f \in G$. We remark that the Yamabe problem is the special case of Conjecture 1.1 when $G=\left\{\operatorname{id}_{M}\right\}$.

Recall that the Yamabe constant of $(M, g)$ is defined as

$$
Y(M, g)=\inf \left\{E(u): 0<u \in C^{\infty}(M)\right\},
$$

where

$$
E(u)=\frac{\int_{M}\left(\frac{4(n-1)}{n-2}\left|\nabla_{g} u\right|^{2}+R_{g} u^{2}\right) d V_{g}}{\left(\int_{M} u^{\frac{2 n}{n-2}} d V_{g}\right)^{\frac{n-2}{n}}} .
$$

In [10], Hebey and Vaugon proved the following:

Theorem 1.2 (Theorem B in [10]) There always holds

$$
\inf \{E(u): u \text { is } G \text {-invariant }\} \leq Y\left(S^{n}, g_{S^{n}}\right)\left(\inf _{x \in M} \operatorname{card} O_{G}(x)\right)^{\frac{2}{n}},
$$

where $O_{G}(x)$ denotes the orbit of $x \in M$ under $G$, i.e. $O_{G}(x)=\{\sigma(x): \sigma \in G\}$, and $Y\left(S^{n}, g_{S^{n}}\right)$ is the Yamabe constant of the standard $n$-dimensional unit sphere $\left(S^{n}, g_{S^{n}}\right)$.

Theorem 1.3 (Theorem $C$ in [10]) If the strict inequality holds in (1.3), then the infimum on the left hand side is achieved and the equivariant Yamabe problem is solvable.

On the other hand, Hebey and Vaugon proved in [10, Theorem B] that the strict inequality holds in (1.3), and as a result the Conjecture 1.1 holds, if one of the following conditions is satisfied:

(i) The action of $G$ on $M$ is free.

(ii) $3 \leq \operatorname{dim} M \leq 11$.

(iii) There exists a point $p \in M$ with finite minimal orbit under $G$ such that $\omega(p):=\inf \{i \in$ $\left.\mathbb{N}:\left\|\nabla^{i} W_{g}(p)\right\| \neq 0\right\}>(n-6) / 2$ or $\omega(p) \in\{0,1,2\}$.

See also $[11,14,15,19]$ for results related to the equivariant Yamabe problem.

The Yamabe problem can be formulated for manifolds with boundary. Let $M$ be a compact $n$-dimensional manifold with smooth boundary $\partial M$, where $n \geq 3$, and $g$ is a Riemannian metric on $M$. There are two types for the Yamabe problem with boundary:

The Yamabe problem with boundary (I). Find a conformal metric $\widetilde{g}$ such that its scalar curvature $R_{\widetilde{g}}$ is constant in $M$ and its mean curvature $H_{\tilde{g}}$ is zero on $\partial M$.

The Yamabe problem with boundary (II). Find a conformal metric $\tilde{g}$ such that its scalar curvature $R_{\widetilde{g}}$ is zero in $M$ and its mean curvature $H_{\widetilde{g}}$ is constant on $\partial M$.

These problems have been studied by many authors. See $[1,5,7-9,16]$ and the references therein.

Inspired by Conjecture 1.1, we study in this paper the equivariant Yamabe problem with boundary. For a compact Riemannian manifold with boundary $(M, \partial M, g)$, the group of conformal transformations $C(M, \partial M, g)$ and the group of isometries $I(M, \partial M, g)$ are respectively defined as

$$
\begin{aligned}
C(M, \partial M, g) & =\left\{f: \bar{M} \rightarrow \bar{M} \text { is diffeomorphism } \mid f^{*} g \in[g]\right\}, \\
I(M, \partial M, g) & =\left\{f: \bar{M} \rightarrow \bar{M} \text { is diffeomorphism } \mid f^{*} g=g\right\} .
\end{aligned}
$$


Note that if $f: \bar{M} \rightarrow \bar{M}$ is diffeomorphism, then $f$ maps $\partial M$ diffeomorphically to $\partial M$, i.e. $\left.f\right|_{\partial M}: \partial M \rightarrow \partial M$ is diffeomorphism. Therefore, if $f \in C(M, \partial M, g)$, then $\left.f\right|_{\partial M} \in$ $C\left(\partial M,\left.g\right|_{\partial M}\right)$. Similarly, if $f \in I(M, \partial M, g)$, then $\left.f\right|_{\partial M} \in I\left(\partial M,\left.g\right|_{\partial M}\right)$.

We are interested in the following two conjectures which are the corresponding versions of the Hebey-Vaugon Conjecture on manifolds with boundary:

Conjecture 1.4 (Equivariant Yamabe problem with boundary (I)) Given a compact subgroup $G$ of $I(M, \partial M, g)$, there exists a $G$-invariant metric conformal to $g$ such that its scalar curvature is constant and its mean curvature vanishes.

Conjecture 1.5 (Equivariant Yamabe problem with boundary(II)) Given a compact subgroup $G$ of $I\left(\partial M,\left.g\right|_{\partial M}\right)$, there exists a $G$-invariant metric conformal to $g$ such that its scalar curvature vanishes and its mean curvature is constant.

We remark that the Yamabe problem with boundary (I) is the special case of Conjecture 1.4 when $G=\left\{\operatorname{id}_{M}\right\}$ and the Yamabe problem with boundary (II) is the special case of Conjecture 1.5 when $G=\left\{\operatorname{id}_{\partial M}\right\}$. We also remark that one may modify Conjecture 1.5 as follows:

Conjecture 1.6 Given a compact subgroup $G$ of $I(M, \partial M, g)$, there exists a $G$-invariant metric conformal to $g$ such that its scalar curvature vanishes and its mean curvature is constant.

Since $f \in I(M, \partial M, g)$ implies that $\left.f\right|_{\partial M} \in I\left(\partial M,\left.g\right|_{\partial M}\right)$, Conjecture 1.6 in fact implies Conjecture 1.5. But we find that it is easier to consider Conjecture 1.5 than the more restrictive Conjecture 1.6.

In this paper, we prove some results related to the equivariant Yamabe problem with boundary (I). To state our results, we define

$$
E(u)=\frac{\int_{M}\left(\frac{4(n-1)}{n-2}\left|\nabla_{g} u\right|^{2}+R_{g} u^{2}\right) d V_{g}+2 \int_{\partial M} H_{g} u^{2} d A_{g}}{\left(\int_{M} u^{\frac{2 n}{n-2}} d V_{g}\right)^{\frac{n-2}{n}}} .
$$

The Yamabe constant with boundary of $(M, \partial M, g)$ is defined as

$$
Y(M, \partial M, g)=\inf \left\{E(u): 0<u \in C^{\infty}(\bar{M})\right\} .
$$

Let $S_{+}^{n}$ be the $n$-dimensional upper hemisphere i.e.

$$
S_{+}^{n}=\left\{x \in \mathbb{R}^{n+1}:|x|^{2}=1, x_{n+1} \geq 0\right\} .
$$

Let $g_{S_{+}^{n}}$ be the standard metric in $S_{+}^{n}$, and the boundary of $S_{+}^{n}$ is given by

$$
\partial S_{+}^{n}=\left\{x \in \mathbb{R}^{n+1}:|x|^{2}=1, x_{n+1}=0\right\} .
$$

The following is the corresponding version of Theorem 1.2:

Theorem 1.7 There always holds

$$
\inf \{E(u): u \text { is } G \text {-invariant }\} \leq Y\left(S_{+}^{n}, \partial S_{+}^{n}, g_{S_{+}^{n}}\right)\left(\inf _{x \in \bar{M}} \operatorname{card} O_{G}(x)\right)^{\frac{2}{n}}
$$

where $O_{G}(x)$ denotes the orbit of $x \in \bar{M}$ under $G$, i.e. $O_{G}(x)=\{\sigma(x): \sigma \in G\}$.

The following is the corresponding version of Theorem 1.3: 
Theorem 1.8 If the strict inequality holds in (1.6), then the infimum on the left hand side is achieved and the equivariant Yamabe problem with boundary (I) is solvable.

We are able to prove that the inequality in (1.6) is strict for some cases. More precisely, we have the following:

Theorem 1.9 Let $(M, g)$ be a compact $n$-dimensional Riemannian manifold with boundary $\partial M$. Assume that $\partial M$ is umbilic and that $M$ is not conformally diffeomorphic to $S_{+}^{n}$. If either $M$ is locally conformally flat or $3 \leq n \leq 5$, then the strict inequality holds in (1.6). In particular, equivariant Yamabe problem with boundary (I) is solvable for such $(M, \partial M, g)$.

Similar results are also obtained for the equivariant Yamabe problem with boundary (II). Define

$$
Q(u)=\frac{\int_{M}\left(\frac{4(n-1)}{n-2}\left|\nabla_{g} u\right|^{2}+R_{g} u^{2}\right) d V_{g}+2 \int_{\partial M} H_{g} u^{2} d A_{g}}{\left(\int_{\partial M} u^{\frac{2(n-1)}{n-2}} d A_{g}\right)^{\frac{n-2}{n-1}}} .
$$

The generalized Yamabe constant of $(M, \partial M, g)$ is defined as

$$
Q(M, \partial M, g)=\inf \left\{Q(u): 0<u \in C^{\infty}(\bar{M})\right\} .
$$

Let $D^{n}$ be the $n$-dimensional unit disk, i.e.

$$
D^{n}=\left\{x \in \mathbb{R}^{n+1}:|x|^{2} \leq 1\right\} .
$$

Then $D^{n}$ equipped with the standard flat metric $g_{D^{n}}$ is an $n$-dimensional Riemannian manifold with boundary

$$
\partial D^{n}=\left\{x \in \mathbb{R}^{n+1}:|x|^{2}=1\right\} .
$$

Similar to Theorem 1.7, we have the following:

Theorem 1.10 There always holds

$$
\inf \left\{Q(u):\left.u\right|_{\partial M} \text { is G-invariant }\right\} \leq Q\left(D^{n}, \partial D^{n}, g_{D^{n}}\right)\left(\inf _{x \in \partial M} \operatorname{card} O_{G}(x)\right)^{\frac{1}{n-1}} .
$$

On the other hand, we can prove the following theorem similar to Theorem 1.8.

Theorem 1.11 If the strict inequality holds in (1.9), then the infimum on the left hand side is achieved and the equivariant Yamabe problem with boundary (II) is solvable.

Similar to Theorem 1.9, we are able to prove that the inequality (1.9) is strict for some cases.

Theorem 1.12 Let $\left(M^{n}, g\right)$ be a compact Riemannian manifold with boundary. Assume that $\partial M$ is umbilic and that $M$ is not conformally diffeomorphic to $D^{n}$. Then the strict inequality holds in (1.9), if either $M$ is locally conformally flat or $3 \leq n \leq 5$. In particular, equivariant Yamabe problem with boundary (II) is solvable for such $(M, \partial M, g)$.

Note that in both Theorem 1.9 and Theorem 1.12, we only considered the case when the boundary $\partial M$ is umbilic. It would be very interesting to consider the equivariant Yamabe problem with boundary in the nonumbilic case, in view of the fact that, for the Escobar's Yamabe problem with boundary, the nonumbilic case is considered to be easier that the umbilic case. We hope that we could return to this in future. 
The plan of this paper is as follows: In Sect. 2, we prove some basic facts related to the equivariant Yamabe problem with boundary (I), and then prove Theorem 1.7 and Theorem 1.8. In Sect. 3, we study the locally conformally flat case of Theorem 1.9. In Sect. 4, we prove the remaining cases of Theorem 1.9, namely, when $3 \leq \operatorname{dim} M \leq 5$. In Sect. 5, after proving some basic facts related to the equivariant Yamabe problem with boundary (II), we then prove Theorem 1.10 and Theorem 1.11. The locally conformally flat case of Theorem 1.12 is proved in Sect. 6 , while the case of Theorem 1.12 when $3 \leq \operatorname{dim} M \leq 5$ is proved in Sect. 7.

\section{Notations and preliminary results for type (I)}

Let $(M, \partial M, g)$ be a compact $n$-dimensional Riemannian manifold with smooth boundary $\partial M$. By conformal change of $g$, we may assume that $H_{g}=0$ on $\partial M$ (see Lemma 1.1 in [9]). It is known that if $Y(M, \partial M, g) \leq 0$, then the Equivariant Yamabe problem with boundary (I) is always solvable; for one can seek the solution to the subcritical case, pass to the limit and get the solution to the critical case, as done by Trudinger for the classical Yamabe problem. More precisely, for all $2<q \leq \frac{2 n}{n-2}$, there exists $u_{q}>0$ such that $u_{q}$ is $G$-invariant and satisfies

$$
\frac{4(n-1)}{n-2} \Delta_{g} u_{q}+R_{g} u_{q}=\mu_{q}(G) u_{q}^{q-1} \text { in } M, \quad \frac{\partial u_{q}}{\partial v_{g}}=0 \text { on } \partial M,
$$

where $\mu_{q}(G)$ is defined below. By the assumption $Y(M, \partial M, g) \leq 0, u_{q}$ converges to $u$ as $q \rightarrow \frac{2 n}{n-2}$, where $u$ is a solution to the Equivariant Yamabe problem with boundary (I).

Hence, we assume that $Y(M, \partial M, g)>0$. By conformal change of $g$, we may assume without loss of generality (see Lemma 1.1 in [9]), i.e.

$$
R_{g}>0 \text { in } M \text { and } H_{g}=0 \text { on } \partial M .
$$

Let

$$
\Omega(M)=\left\{u \in H_{1}^{2}(M): \frac{\partial u}{\partial \nu_{g}}=0 \text { on } \partial M\right\} .
$$

It follows from Sobolev embedding that $\Omega(M) \subseteq L^{q}(M)$ for $2 \leq q \leq \frac{2 n}{n-2}$.

For $2 \leq q \leq \frac{2 n}{n-2}$, we define

$$
E_{q}(u)=\frac{\int_{M}\left(\frac{4(n-1)}{n-2}\left|\nabla_{g} u\right|^{2}+R_{g} u^{2}\right) d V_{g}}{\left(\int_{M} u^{q} d V_{g}\right)^{\frac{2}{q}}}
$$

for $0 \not \equiv u \in \Omega(M)$. In view of (2.1), we have $E_{\frac{2 n}{n-2}}(u)=E(u)$ defined in (1.4). For $G \subset I(M, \partial M, g)$ and $2 \leq q \leq \frac{2 n}{n-2}$, we define

$$
\mu_{q}(G)=\inf \left\{E_{q}(u): 0 \not \equiv u \in \Omega(M) \text { and } u \text { is } G \text {-invariant }\right\} \text {, }
$$

which is well-defined, since $\Omega(M) \subseteq L^{q}(M)$ for any $2 \leq q \leq \frac{2 n}{n-2}$ (see Theorem 2.30 in [3] for example). 
Lemma 2.1 For all $2 \leq q<\frac{2 n}{n-2}$ and all $G \subseteq I(M, \partial M, g), \mu_{q}(G)$ is attained. Thus there exists $0<u_{q} \in C^{\infty}(\bar{M})$ which is $G$-invariant such that

$$
\frac{4(n-1)}{n-2} \Delta_{g} u_{q}+R_{g} u_{q}=\mu_{q}(G) u_{q}^{q-1} \text { in } M, \quad \frac{\partial u_{q}}{\partial v_{g}}=0 \text { on } \partial M, \quad \int_{M} u_{q}^{q} d V_{g}=1 .
$$

Proof Consider a sequence $\left\{u_{i}\right\}$ of positive functions in $\Omega(M)$ which is $G$-invariant and satisfies

$$
\lim _{i \rightarrow \infty} E_{q}\left(u_{i}\right)=\mu_{q}(G) \text { and } \int_{M} u_{i}^{q} d V_{g}=1
$$

Since the embedding $\Omega(M) \hookrightarrow L^{q}(M)$ is compact for all $2 \leq q<\frac{2 n}{n-2}$ (c.f. Theorem 2.34 in [3]), by passing to a subsequence, we can suppose

$$
\begin{aligned}
& \lim _{i \rightarrow \infty} u_{i}=u_{q} \text { strongly in } L^{2}(M) \cap L^{q}(M), \\
& \lim _{i \rightarrow \infty} u_{i}=u_{q} \text { weakly in } H_{1}^{2}(M), \text { and } \\
& \lim _{i \rightarrow \infty} u_{i}=u_{q} \text { almost everywhere. }
\end{aligned}
$$

By (2.5), $u_{q}$ is positive almost everywhere. Since $u_{i}$ is $G$-invariant, we have $u_{i}(\gamma x)=$ $u_{i}(x)$ for all $\gamma \in G$ and all $x \in \bar{M}$, which together with (2.5) implies that $u_{q}(\gamma x)=$ $\lim _{i \rightarrow \infty} u_{i}(\gamma x)=\lim _{i \rightarrow \infty} u_{i}(x)=u_{q}(x)$, i.e. $u_{q}$ is $G$-invariant. By (2.2) and (2.3), we have

$$
1=\lim _{i \rightarrow \infty} \int_{M} u_{i}^{q} d V_{g}=\int_{M} u_{q}^{q} d V_{g} \text { and } \lim _{i \rightarrow \infty} \int_{M} R_{g} u_{i}^{2} d V_{g}=\int_{M} R_{g} u_{q}^{2} d V_{g} .
$$

We compute

$$
\begin{aligned}
0 & \leq \int_{M}\left|\nabla_{g} u_{i}-\nabla_{g} u_{q}\right|^{2} d V_{g} \\
& =\int_{M}\left|\nabla_{g} u_{i}\right|^{2} d V_{g}+\int_{M}\left|\nabla_{g} u_{q}\right|^{2} d V_{g}-2 \int_{M}\left\langle\nabla_{g} u_{i}, \nabla_{g} u_{q}\right\rangle d V_{g} .
\end{aligned}
$$

It follows from (2.4) that

$$
\lim _{i \rightarrow \infty} \int_{M}\left\langle\nabla_{g} u_{i}, \nabla_{g} u_{q}\right\rangle d V_{g}=\int_{M}\left|\nabla_{g} u_{q}\right|^{2} d V_{g} .
$$

Combining this with (2.7), we obtain

$$
\lim _{i \rightarrow \infty} \int_{M}\left|\nabla_{g} u_{i}\right|^{2} d V_{g} \geq \int_{M}\left|\nabla_{g} u_{q}\right|^{2} d V_{g} .
$$

By (2.1), (2.2), (2.6) and (2.8), we have

$$
\begin{aligned}
E_{q}\left(u_{q}\right) & =\int_{M}\left(\frac{4(n-1)}{n-2}\left|\nabla_{g} u_{q}\right|^{2}+R_{g} u_{q}^{2}\right) d V_{g} \\
& \leq \lim _{i \rightarrow \infty} \int_{M}\left(\frac{4(n-1)}{n-2}\left|\nabla_{g} u_{i}\right|^{2}+R_{g} u_{i}^{2}\right) d V_{g} \\
& =\lim _{i \rightarrow \infty} E_{q}\left(u_{i}\right)=\mu_{q}(G) .
\end{aligned}
$$


This implies that $u_{q}$ realizes the minimum of $E_{q}(u)$ in the space of $u \in \Omega(M)$ which are $G$-invariant. The Euler-Lagrange equation then implies that

$$
\int_{M}\left(\frac{4(n-1)}{n-2}\left\langle\nabla_{g} u_{q}, \nabla_{g} v\right\rangle+R_{g} u_{q} v\right) d V_{g}=\mu_{q}(G) \int_{M} u_{q}^{q-1} v d V_{g}
$$

for all $G$-invariant functions $v \in \Omega(M)$. It also follows from (2.4) that

$$
\frac{\partial u_{q}}{\partial v_{g}}=\lim _{i \rightarrow \infty} \frac{\partial u_{i}}{\partial v_{g}}=0 \text { on } \partial M
$$

Let $L_{g}$ be the conformal Laplacian of $g$ :

$$
L_{g}(u)=\frac{4(n-1)}{n-2} \Delta_{g} u+R_{g} u \text { for } u \in \Omega(M) .
$$

Since $R_{g}>0$ by (2.1), the conformal Laplacian $L_{g}$ defined in $\Omega(M)$ is invertible. Hence, let $w \in \Omega(M)$ be the unique solution of the equation

$$
L_{g}(w)=\mu_{q}(G) u_{q}^{q-1} .
$$

Since $u_{q}$ is $G$-invariant, the uniqueness of $w$ implies that $w$ is also $G$-invariant. On the other hand, it follows from (2.9) and (2.11) that

$$
\int_{M}\left(\frac{4(n-1)}{n-2}\left\langle\nabla_{g}\left(u_{q}-w\right), \nabla_{g} v\right\rangle+R_{g}\left(u_{q}-w\right) v\right) d V_{g}=0
$$

for all $G$-invariant functions $v$ in $\Omega(M)$. Since $u_{q}-w$ is $G$-invariant, we have

$$
\begin{aligned}
0 & =\int_{M}\left(\frac{4(n-1)}{n-2}\left|\nabla_{g}\left(u_{q}-w\right)\right|^{2}+R_{g}\left(u_{q}-w\right)^{2}\right) d V_{g} \\
& =\int_{M}\left(u_{q}-w\right) L_{g}\left(u_{q}-w\right) d V_{g}
\end{aligned}
$$

where we have used (2.10) and integration by parts. Since $L_{g}$ is invertible, $u_{q}=w$, i.e. $u_{q}$ is a weak solution of

$$
L_{g}\left(u_{q}\right)=\mu_{q}(G) u_{q}^{q-1} \text { in } M \text { and } \frac{\partial u_{q}}{\partial v_{g}}=0 \text { on } \partial M .
$$

A regularity theorem of Cherrier [6] asserts that $u_{q} \in C^{\infty}(\bar{M})$. This proves the assertion.

Lemma 2.2 If a subsequence of the sequence $\left\{u_{q}\right\}_{2 \leq q<\frac{2 n}{n-2}}$ converges strongly in $L^{2}(M)$ to a function $0 \not \equiv u \in \Omega(M)$, then $u$ realizes the minimum of $E_{\frac{2 n}{n-2}}$ in the space of functions in $\Omega(M)$ which are $G$-invariant. In particular,

(i) $u$ is a positive smooth $G$-invariant function in $M$,

(ii) $\frac{\partial u}{\partial v_{g}}=0$ on $\partial M$ and $\frac{4(n-1)}{n-2} \Delta_{g} u+R_{g} u=\mu_{\frac{2 n}{n-2}}(G) u^{\frac{n+2}{n-2}}$ in $M$, and

(iii) $\int_{M} u^{\frac{2 n}{n-2}} d V_{g}=1$.

Proof First of all, we note that

$$
\limsup _{q \rightarrow \frac{2 n}{n-2}} \mu_{q}(G) \leq \mu_{\frac{2 n}{n-2}}(G) .
$$


To see this, it suffices to consider $0<v \in C^{\infty}(\bar{M})$ which is $G$-invariant and satisfies $E_{\frac{2 n}{n-2}}(v) \leq \mu_{\frac{2 n}{n-2}}(G)+\epsilon$. As $\mu_{q}(G) \leq E_{q}(v)$ with $\lim _{q \rightarrow \frac{2 n}{n-2}} E_{q}(v)=E_{\frac{2 n}{n-2}}(v)$, we get

$$
\lim _{q \rightarrow \frac{2 n}{n-2}} \mu_{q}(G) \leq \lim _{q \rightarrow \frac{2 n}{n-2}} E_{q}(v)=E_{\frac{2 n}{n-2}}(v) \leq \mu_{\frac{2 n}{n-2}}(G)+\epsilon .
$$

Without loss of generality, we suppose now that the subsequence $\left\{u_{q}\right\}$ satisfies $\lim u_{q}=u$ strongly in $L^{2}(M) \cap L^{\frac{2 n}{n-2}}(M)$, weakly in $\Omega(M)$ and almost everywhere with

$$
\mu=\lim \mu_{q}(G) \leq \mu_{\frac{2 n}{n-2}}(G) .
$$

By reasoning as in the proof of Lemma 2.1, we see that $0<u \in C^{\infty}(\bar{M})$ is $G$-invariant and

$$
\frac{\partial u}{\partial v_{g}}=0 \text { on } \partial M \text { and } \frac{4(n-1)}{n-2} \Delta_{g} u+R_{g} u=\mu u^{\frac{n+2}{n-2}} \text { in } M .
$$

It remains to show that $\mu=\mu_{\frac{2 n}{n-2}}(G)$ and $\int_{M} u^{\frac{2 n}{n-2}} d V_{g}=1$. We find

$$
\begin{aligned}
\int_{M} u^{\frac{2 n}{n-2}} d V_{g} & =\lim _{q \rightarrow \frac{2 n}{n-2}} \int_{M} u_{q}^{\frac{n+2}{n-2}} u d V_{g} \\
& \leq \lim _{q \rightarrow \frac{2 n}{n-2}}\left(\int_{M} u_{q}^{q} d V_{g}\right)^{\frac{n+2}{(n-2) q}}\left(\int_{M} u^{\frac{(n-2) q}{(n-2) q-(n+2)}} d V_{g}\right)^{1-\frac{n+2}{(n-2) q}} \\
& \leq\left(\int_{M} u^{\frac{2 n}{n-2}} d V_{g}\right)^{\frac{n-2}{2 n}}
\end{aligned}
$$

where the second inequality follows from $\int_{M} u_{q}^{q} d V_{g}=1$, which implies that

$$
\int_{M} u^{\frac{2 n}{n-2}} d V_{g} \leq 1
$$

Multiplying the second equation in (2.13) by $u$, integrating over $M$ and using the first equation in (2.13), we get

$$
\mu_{\frac{2 n}{n-2}}(G) \leq E_{\frac{2 n}{n-2}}(u)=\mu\left(\int_{M} u^{\frac{2 n}{n-2}} d V_{g}\right)^{\frac{2}{n}} \leq \mu,
$$

where the last inequality follows from (2.14). Combining (2.12), (2.14) and (2.15), we can conclude that $\mu=\mu_{\frac{2 n}{n-2}}(G)$ and $\int_{M} u^{\frac{2 n}{n-2}} d V_{g}=1$, as required.

It now remains to study the case when Lemma 2.2 does not happen. So for any subsequence of $\left\{u_{q}\right\}_{2 \leq q<\frac{2 n}{n-2}}$, which converges in $L^{p}(M)$ with $2 \leq p \leq \frac{2 n}{n-2}$, converges to zero. This phenomena occurs when $\left\{u_{q}\right\}$ has concentration points.

For $r>0$ and $x \in \bar{M}$, set $B_{r}(x)=\left\{y \in \bar{M}: d_{g}(x, y)<r\right\}$. Here, $d_{g}(x, y)$ is the distance between $x$ and $y$ in $\bar{M}$ with respect to the Riemannian metric $g$.

Lemma 2.3 In the situation described above, there exists a finite number of points $\left\{x_{1}, \cdots, x_{k}\right\}$ in $\bar{M}$ for which 


$$
\inf _{G} E(u)\left(\limsup _{q \rightarrow \frac{2 n}{n-2}} \int_{B_{\delta}\left(x_{i}\right) \cap M} u_{q}^{q} d V_{g}\right)^{2 / n} \geq Y\left(S_{+}^{n}, \partial S_{+}^{n}, g_{S_{+}^{n}}\right)
$$

for all $\delta>0$ and for each $i=1,2 \ldots, k$.

The proof of Lemma 2.3 will be given in Appendix. Now we are ready to prove Theorem 1.8 .

Proof of Theorem 1.8 By the assumption in Theorem 1.8, $\mu_{\frac{2 n}{n-2}}(G)$ is strictly smaller than $Y\left(S_{+}^{n}, \partial S_{+}^{n}, g_{S_{+}^{n}}\right)\left(\inf _{x \in \bar{M}} \text { card } O_{G}(x)\right)^{\frac{2}{n}}$ and it is about to show that the situation described above cannot occur (so that Lemma 2.2 applies).

Suppose for any subsequence of $\left\{u_{q}\right\}_{2 \leq q<\frac{2 n}{n-2}}$, which converges in $L^{p}(M)$ with $2 \leq p \leq$ $\frac{2 n}{n-2}$, converges to zero. By Lemma 2.3 , there exist a finite numbers of points $\left\{x_{1}, \cdots, x_{k}\right\}$ for which for all $\delta>0$

$$
\mu_{\frac{2 n}{n-2}}(G)\left(\limsup _{q \rightarrow \frac{2 n}{n-2}} \int_{B_{\delta}\left(x_{i}\right) \cap M} u_{q}^{q} d V_{g}\right)^{\frac{2}{n}} \geq Y\left(S_{+}^{n}, \partial S_{+}^{n}, g_{S_{+}^{n}}\right) .
$$

We now use the invariance of $u_{q}$ under $G$ and the property

$$
\int_{M} u_{q}^{q} d V_{g}=1
$$

Two cases arise. In the first case, the orbit of $x_{i}$ in $G$ is finite, i.e. card $O_{G}\left(x_{i}\right)$ is finite. It follows from (2.17) that we can choose $\delta>0$ small enough to get $\int_{B_{\delta}\left(x_{i}\right) \cap M} u_{q}^{q} d V_{g} \leq$ $\left(\operatorname{card} O_{G}\left(x_{i}\right)\right)^{-1}$, which gives

$$
\limsup _{q \rightarrow \frac{2 n}{n-2}} \int_{B_{\delta}\left(x_{i}\right) \cap M} u_{q}^{q} d V_{g} \leq\left(\operatorname{card} O_{G}\left(x_{i}\right)\right)^{-1} .
$$

In the second case, card $O_{G}\left(x_{i}\right)=\infty$. It is then easy to see that, for all $\epsilon>0$, there exists $0<\delta \ll 1$ with $\limsup _{q \rightarrow \frac{2 n}{n-2}} \int_{B_{\delta}\left(x_{i}\right) \cap M} u_{q}^{q} d V_{g} \leq \epsilon$. But if $\epsilon$ is sufficiently small, this equality contradicts (2.16).

Therefore, for all $i=1, \ldots, k$, the orbit of $x_{i}$ in $G$ is finite and

$$
\mu_{\frac{2 n}{n-2}}(G) \geq Y\left(S_{+}^{n}, \partial S_{+}^{n}, g_{S_{+}^{n}}\right)\left(\operatorname{card} O_{G}\left(x_{i}\right)\right)^{\frac{2}{n}}
$$

by (2.16) and (2.18). The hypothesis that the strict inequality holds in (1.6), i.e.

$$
\mu_{\frac{2 n}{n-2}}(G)<Y\left(S_{+}^{n}, \partial S_{+}^{n}, g_{S_{+}^{n}}\right)\left(\inf _{x \in \bar{M}} \operatorname{card} O_{G}(x)\right)^{\frac{2}{n}}
$$

contradicts to (2.19). Therefore, $x_{i}$ in fact does not exist, and $\left\{u_{q}\right\}_{2 \leq q<\frac{2 n}{n-2}}$ satisfies the conditions in Lemma 2.2. This proves Theorem 1.8. 
We remark that we just showed that if the orbit of any point in $G$ is infinite, then $\mu_{\frac{2 n}{n-2}}(G)$ is attained.

Proof of Theorem 1.7 If the orbit of any point in $G$ is infinite, there is nothing to prove. Suppose that $G \subseteq I(M, \partial M, g)$ possesses a finite orbit. Let $x_{1}$ be a point of minimal orbit.

Consider the case when $x_{1} \in M$. If $x_{1} \in M$, then by conformally changing $g$, we may assume that the mean curvature of $g$ vanishes and the scalar curvature of $g$ at $x_{1}$ is zero, i.e. $H_{g}=0$ on $\partial M$ and $R_{g}\left(x_{1}\right)=0$. To see this, suppose that $f \in C^{\infty}(\bar{M})$ such that $f$ is $G$-invariant and is strictly positive in at least one point of $\bar{M}$ which satisfies $f\left(x_{1}\right)=0$. Since the orbit of $x_{1}$ in $G$ is finite, it is always possible to construct such a function $f$. Let $0<u \in C^{\infty}(\bar{M})$ be the unique solution of

$$
\frac{\partial u}{\partial v_{g}}=0 \text { on } \partial M \text { and } L_{g}(u)=f u^{q} \text { in } M
$$

where $2 \leq q<\frac{2 n}{n-2}$. Since $f$ is $G$-invariant, $u$ is also $G$-invariant. Then the conformal metric $\widetilde{g}=u^{\frac{4}{n-2}} g$ satisfies

$$
\begin{aligned}
& H_{\tilde{g}}=u^{-\frac{n}{n-2}}\left(\frac{\partial u}{\partial \nu_{g}}+H_{g} u\right)=0 \text { on } \partial M, \\
& R_{\widetilde{g}}=u^{-\frac{n+2}{n-2}} L_{g} u=f u^{q-\frac{n+2}{n-2}} \text { in } M
\end{aligned}
$$

by (2.1) and (2.20). In particular, we have $R_{\widetilde{g}}\left(x_{1}\right)=f\left(x_{1}\right) u\left(x_{1}\right)^{q-\frac{n+2}{n-2}}=0$, as required.

Now let $O_{G}\left(x_{1}\right)=\left\{x_{1}, \ldots, x_{k}\right\}$. Let $\delta>0$ be chosen such that $B\left(x_{i}, \delta\right) \cap B\left(x_{j}, \delta\right)=\varnothing$ if $i \neq j$ and $B\left(x_{i}, \delta\right) \cap \partial M=\varnothing$. For $i=1, \ldots, k$, we define

$$
u_{i, \epsilon}(x)= \begin{cases}\left(\epsilon+d\left(x_{i}, x\right)^{2}\right)^{1-n / 2}-\left(\epsilon+\delta^{2}\right)^{1-n / 2}, & \text { if } d\left(x_{i}, x\right) \leq \delta \\ 0, & \text { otherwise }\end{cases}
$$

and let $u_{\epsilon}=\sum_{i=1}^{k} u_{i, \epsilon}$. The function $u_{\epsilon}$ is therefore $G$-invariant and since $E_{\frac{2 n}{n-2}}\left(u_{\epsilon}\right)=$ $k^{\frac{2}{n}} E_{\frac{2 n}{n-2}}\left(u_{1, \epsilon}\right)$, we can deduce that

$$
\lim _{\epsilon \rightarrow 0} E_{\frac{2 n}{n-2}}\left(u_{\epsilon}\right)=k^{\frac{2}{n}} Y\left(S_{+}^{n}, \partial S_{+}^{n}, g_{S_{+}^{n}}\right)
$$

which implies (1.6) since $k=\operatorname{card} O_{G}\left(x_{1}\right)=\inf _{x \in \bar{M}} \operatorname{card} O_{G}(x)$.

Let $x_{1} \in \partial M$ be the point of minimal orbit of $\partial M$ under $G$, i.e. $k:=\operatorname{card} O_{G}\left(x_{1}\right)=$ inf card $O_{G}(x)$. Then we can construct a Riemannian metric $g^{\prime}$ conformal to $g$ and $G$ $x \in \bar{M}$ invariant which satisfies $R c\left(g^{\prime}\right)=0$ and $H\left(g^{\prime}\right)=0$ at any point of a minimal orbit under $G$ (See Lemma 4.1).

Let $\left(y_{1}^{1}, \cdots, y_{n}^{1}\right)$ be normal coordinates around $x_{1} \in \partial M$, such that $v_{g}\left(x_{1}\right)=-\frac{\partial}{\partial y_{n}^{1}}$ and the second fundamental form of $\partial M$ at $x_{1}$ has a diagonal form. By the invariance under $G$, we have normal coordinates $\left(y_{1}^{i}, \cdots, y_{n}^{i}\right)$ around each $x_{i} \in O_{G}\left(x_{1}\right)$ which satisfy the properties described above. Let $\delta>0$ be chosen such that $B\left(x_{i}, \delta\right) \cap B\left(x_{j}, \delta\right)=\varnothing$ if $i \neq j$. Let $s_{i}(x)=\max \left\{\left|y^{i}\right|,\left|y_{n}^{i}\right|\right\}$, where $\left|y^{i}\right|^{2}=\left(y_{1}^{i}\right)^{2}+\cdots+\left(y_{n-1}^{i}\right)^{2}$, and $\psi(s)$ be a piecewise smooth function which satisfies $\psi(s)=1$ for $|s| \leq \delta, \psi(s)=0$ for $|s| \geq 2 \delta$, and $\left|\psi^{\prime}(s)\right| \leq 2 \delta^{-1}$ for $\delta \leq|s| \leq 2 \delta$. For $i=1, \ldots, k$, we define

$$
u_{i, \epsilon}=v_{i}\left(\psi \circ s_{i}\right)
$$


where

$$
v_{i}=\left(\frac{\epsilon}{\epsilon^{2}+\left|y^{i}\right|^{2}-\delta\left(y_{n}^{i}\right)^{2}}\right)^{\frac{n-2}{2}}
$$

and let $u_{\epsilon}=\sum_{i=1}^{k} u_{i, \epsilon}$. The function $u_{\epsilon}$ is therefore $G$-invariant and since $E_{\frac{2 n}{n-2}}\left(u_{\epsilon}\right)=$ $k^{\frac{2}{n}} E_{\frac{2 n}{n-2}}\left(u_{1, \epsilon}\right)$, we can deduce that (see P. 32-33 in [9])

$$
\lim _{\epsilon \rightarrow 0} E_{\frac{2 n}{n-2}}\left(u_{\epsilon}\right)=k^{\frac{2}{n}} \lim _{\epsilon \rightarrow 0} E_{\frac{2 n}{n-2}}\left(u_{1, \epsilon}\right) \leq k^{\frac{2}{n}} Y\left(S_{+}^{n}, \partial S_{+}^{n}, g_{S_{+}^{n}}\right),
$$

which implies (1.6) since $k=\operatorname{card} O_{G}\left(x_{1}\right)=\inf _{x \in \bar{M}} \operatorname{card} O_{G}(x)$.

Corollary 2.4 The $x_{i}$ of Lemma 2.3 are necessarily points of minimal orbits under $G$. And in fact we can show that they constitute exactly a minimal orbit of $\bar{M}$ under $G$.

Lemma 2.5 If $x$ is a point of $\overline{S_{+}^{n}}$, we denote by

$$
C_{x}\left(S_{+}^{n}, \partial S_{+}^{n}, g_{S_{+}^{n}}\right)=\left\{\sigma \in C\left(S_{+}^{n}, \partial S_{+}^{n}, g_{S_{+}^{n}}\right): \sigma(x)=x\right\}
$$

and

$$
I_{x}\left(S_{+}^{n}, \partial S_{+}^{n}, g_{S_{+}^{n}}\right)=\left\{\sigma \in I\left(S_{+}^{n}, \partial S_{+}^{n}, g_{S_{+}^{n}}\right): \sigma(x)=x\right\} .
$$

We now consider $g$ a metric on $S_{+}^{n}$ conformal to $g_{S_{+}^{n}}$, and $I_{x}\left(S_{+}^{n}, \partial S_{+}^{n}, g\right)=\{\sigma \in$ $\left.I\left(S_{+}^{n}, \partial S_{+}^{n}, g\right): \sigma(x)=x\right\}$. Then there exists $\tau \in C_{x}\left(S_{+}^{n}, \partial S_{+}^{n}, g_{S_{+}^{n}}\right)$ such that

$$
\tau^{-1} I_{x}\left(S_{+}^{n}, \partial S_{+}^{n}, g\right) \tau \subset I_{x}\left(S_{+}^{n}, \partial S_{+}^{n}, g_{S_{+}^{n}}\right) .
$$

Proof Note that $I_{x}\left(S_{+}^{n}, \partial S_{+}^{n}, g\right)$ is a compact subgroup of conformal transformation of $S_{+}^{n}$. We only deal with the case of $x \in \partial S_{+}^{n}$ here, and the case of $x \in S_{+}^{n}$ can be proved in the same way. Suppose $x \in \partial S_{+}^{n}$. If $\varphi: S_{+}^{n}-\{x\} \rightarrow \mathbb{R}_{+}^{n}$ is the stereographic projection of pole $x$, then $\left.\varphi\right|_{\partial S_{+}^{n}-\{x\}}$ is a map from $\partial S_{+}^{n}$ to $\mathbb{R}^{n-1}$. Then $\left.\varphi\right|_{\partial S_{+}^{n}-\{x\}} I_{x}\left(S_{+}^{n}, \partial S_{+}^{n}, g\right)\left(\left.\varphi\right|_{\partial S_{+}^{n}-\{x\}}\right)^{-1}$ becomes a compact group of conformal transformations of $\mathbb{R}^{n-1}$, a subgroup of the group of isometries of a metric conformal to the Euclidean metric, and thus makes it a subcompact group of the affine group. Consequently, $\left.\varphi\right|_{\partial S_{+}^{n}-\{x\}} I_{x}\left(S_{+}^{n}, \partial S_{+}^{n}, g\right)\left(\left.\varphi\right|_{\partial S_{+}^{n}-\{x\}}\right)^{-1}$ necessarily admits a fixed point (which we denote by $y \in \mathbb{R}^{n-1}=\partial \mathbb{R}_{+}^{n}$ ). Now, if $t_{y}, t_{-y}: \mathbb{R}_{+}^{n} \rightarrow \mathbb{R}_{+}^{n}$ represent the translations of respective vectors $y$ and $-y$, then $t_{-y}\left(\varphi I_{x}\left(S_{+}^{n}, \partial S_{+}^{n}, g\right) \varphi^{-1}\right) t_{y}$ becomes a group of vector isometries of $\mathbb{R}_{+}^{n}$. So, one can see that the conformal transformation $\tau$ we want is given by $\tau=\varphi^{-1} t_{y} \varphi$.

\section{Locally conformally flat case for type (I)}

In this section, we prove the first case of Theorem 1.9, i.e. we will show that if the manifold is locally conformally flat, then the strict inequality holds in (1.6). Let $(M, \partial M, g)$ be a compact Riemannian manifold with boundary and $G \subset I(M, \partial M, g)$ be a subgroup of the group of isometries of $g$. Assume that $\partial M$ is umbilic and that $M$ is not conformally diffeomorphic to $S_{+}^{n}$. We suppose that $G$ has finite orbits, and we denote by $O_{G}\left(x_{1}\right)=\left\{x_{1}, \cdots, x_{k}\right\}$ a minimal orbit under $G$. First, we consider the case of $x_{1} \in \partial M$.

Suppose that $(M, \partial M, g)$ is locally conformally flat. By conformal change of $g$ if necessary, we can assume that $\partial M$ is minimal with respect to $g$ (see Lemma 2.1 in [4]), i.e. $H_{g}=0$. Since $\partial M$ is minimal and umbilic, $\partial M$ is totally geodesic. We have the following: 
Lemma 3.1 If $(M, \partial M, g)$ is locally conformally flat, then for all $x \in \partial M$, there exits a metric $g_{x}^{\prime}$ conformal to $g$ and $I_{x}(M, \partial M, g)$-invariant which is Euclidean in the neighborhood of $x$.

Proof Let $\Omega$ be an open neighborhood of $x$. Since $(M, \partial M, g)$ is locally conformally flat and $\partial M$ is totally geodesic, we can double $M$ along $\partial M$ to obtain $\tilde{M}$, which is locally conformal flat, closed manifold. Then we can a conformal immersion $\tilde{\varphi}$ from a neighborhood of $x \in \tilde{M}$ to $S^{n}$ (c.f. [12]), which gives a conformal immersion $\varphi:(\Omega, g) \rightarrow\left(S_{+}^{n}, g_{S_{+}^{n}}\right)$.

We denote by $p=\varphi(x)$. With Liouville's theorem, $\varphi I_{x}(M, \partial M, g) \varphi^{-1}$ is a compact subgroup of $C_{p}\left(S_{+}^{n}, \partial S_{+}^{n}, g_{S_{+}^{n}}\right)$. Therefore it is also a subgroup of $I_{p}\left(S_{+}^{n}, \partial S_{+}^{n}, g^{\prime}\right)$ for a metric $g^{\prime}$ conformal to $g_{S_{+}^{n}}$. Then, by Lemma 2.5 , there exists $\tau \in C_{p}\left(S_{+}^{n}, \partial S_{+}^{n}, g_{S_{+}^{n}}\right)$ so that $\varphi I_{x}(M, \partial M, g) \varphi^{-1} \subset \tau^{-1} I_{p}\left(S_{+}^{n}, \partial S_{+}^{n}, g_{S_{+}^{n}}\right) \tau$. We set $\psi=\tau \circ \varphi$ and let $g_{0}$ be the Euclidean metric on $S_{+}^{n}-\{p\}$ conformal to $g_{S_{+}^{n}}$, which is obtained by reciprocal image of the Euclidean metric of $\mathbb{R}_{+}^{n}$ under the stereographic projection of pole $-p$. It is easy to see that $g_{0}$ is $I_{p}\left(S_{+}^{n}, \partial S_{+}^{n}, g_{S_{+}^{n}}\right)$-invariant.

We now consider $g_{x}=\psi^{*} g_{0}$. By construction, $g_{0}$ is indeed Euclidean in the neighborhood of $x$. If $\sigma \in I_{x}(M, \partial M, g)$, we have

$$
\begin{aligned}
\sigma^{*} g_{x} & =\sigma^{*}\left(\psi^{*} g_{0}\right) \\
& =(\tau \circ \varphi \circ \sigma)^{*} g_{0} \\
& =\psi^{*}\left(\left(\tau \circ \varphi \circ \sigma \circ \varphi^{-1} \circ \tau^{-1}\right)^{*} g_{0}\right),
\end{aligned}
$$

and, since $\tau \circ \varphi \circ \tau \circ \varphi^{-1} \circ \tau^{-1} \in I_{p}\left(S_{+}^{n}, \partial S_{+}^{n}, g_{S_{+}^{n}}\right)$, we have $\sigma^{*} g_{x}=g_{x}$. So $g_{x}$ is $I_{x}(M, \partial M, g)$-invariant, and setting $g_{x}^{\prime}=\eta g_{x}+(1-\eta) g$ proves the lemma.

Now we consider the metric

$$
g^{\prime}=\sum_{i=1}^{k}\left(\sigma_{i}^{-1}\right)^{*}\left(\eta g_{x_{1}}\right)+\left[\prod_{i=1}^{k}\left(1-\eta \circ \sigma_{i}^{-1}\right)\right] g
$$

where $O_{G}\left(x_{1}\right)=\left\{\sigma_{1}\left(x_{1}\right), \cdots, \sigma_{k}\left(x_{1}\right)\right\}$. Then we can see that the metric $g^{\prime}$ is $G$-invariant and Euclidean in the neighborhood of each point of a minimal orbit under $G$. Then, we can assume that near each $x_{i} \in \partial M$ the metric is flat and the boundary is minimal. Let $\left(y_{1}^{i}, \cdots, y_{n}^{i}\right)$ be rectangular coordinates around $x_{i} \in \partial M$. Since $\partial M$ is umbilic and minimal, it is a hyperplane. So we can assume that $\partial M$ is given by $y_{n}^{i}=0$ in the coordinates $\left(y_{1}^{i}, \cdots, y_{n}^{i}\right)$. Let $G_{i}$ be the positive solution of $L_{g} G_{i}=0$ on $M$ and $\frac{\partial G_{i}}{\partial \nu_{g}}=0$ on $\partial M-\left\{x_{i}\right\}$ where

$$
L(u)=-\frac{4(n-1)}{n-2} \Delta_{g} u+R_{g} u
$$

In the neighborhood of $x_{i}, G_{i}$ admits an expansion of the type

$$
G_{i}(x)=r_{i}^{2-n}+A+\alpha_{i}(x)
$$

where $A$ is a constant, $\alpha_{i}(x)$ is a smooth harmonic function near $x_{i}$ with $\alpha\left(x_{i}\right)=0$, and $r_{i}=d\left(x_{i}, x\right)$. 
If $\sigma\left(x_{i}\right)=x_{j}, G_{j} \circ \sigma=G_{i}$, so that $\sum_{i=1}^{k} G_{i}$ is $G$-invariant. For each $x_{i}$, we now consider the function $u_{\delta, \epsilon}^{i}$ (like [9]) defined by

$$
u_{\delta, \epsilon}^{i}= \begin{cases}\left(\frac{\epsilon}{\epsilon^{2}+r_{i}^{2}}\right)^{(n-2) / 2}, & \text { for } x \in M \cap B_{\delta}\left(x_{i}\right) ; \\ \epsilon_{0}\left(G_{i}(x)-\eta_{i}(x) \alpha_{i}(x)\right), & \text { for } x \in M \cap\left(B_{2 \delta}\left(x_{i}\right)-B_{\delta}\left(x_{i}\right)\right) \\ \epsilon_{0} G_{i}(x), & \text { for } x \in M-B_{2 \delta}\left(x_{i}\right)\end{cases}
$$

where $\delta$ is chosen small enough so that $g$ is Euclidean on $B_{2 \delta}\left(x_{i}\right)$ with $B_{2 \delta}\left(x_{i}\right) \cap B_{2 \delta}\left(x_{j}\right)=\varnothing$ if $i \neq j$, and $\eta_{i}(x)$ is a smooth cut-off function such that $0 \leq \eta_{i} \leq 1, \eta_{i}(x)=1$ if $r_{i} \leq \delta$, $\eta_{i}(x)=0$ if $r_{i} \geq 2 \delta$ and $\left|\nabla_{g} \eta_{i}\right| \leq 2 / \delta$. In order for the function $u_{\delta, \epsilon}^{i}$ to be continuous across $\partial B_{\delta}\left(x_{i}\right)$ we must require $\epsilon_{0}$ to satisfy

$$
\left(\frac{\epsilon}{\epsilon^{2}+\delta^{2}}\right)^{(n-2) / 2}=\epsilon_{0}\left(\delta^{2-n}+A\right)
$$

We set $u_{\delta, \epsilon}=\sum_{i=1}^{k} u_{\delta, \epsilon}^{i}$ so that the function $u_{\delta, \epsilon}$ is $G$-invariant. To simplify the notations, we will write $u=u_{\delta, \epsilon}$ and $u_{i}=u_{\delta, \epsilon}^{i}$. We have

$$
\begin{aligned}
\frac{4(n-1)}{n-2} \int_{M}\left|\nabla_{g} u\right|^{2} d & V_{g}+\int_{M} R_{g} u^{2} d V_{g} \\
= & \sum_{i=1}^{k}\left(\frac{4(n-1)}{n-2} \int_{M}\left|\nabla_{g} u_{i}\right|^{2} d V_{g}+\int_{M} R_{g} u_{i}^{2} d V_{g}\right) \\
& +\sum_{i \neq j}\left(\frac{4(n-1)}{n-2} \int_{M}\left\langle\nabla_{g} u_{i}, \nabla_{g} u_{j}\right\rangle d V_{g}+\int_{M} R_{g} u_{i} u_{j} d V_{g}\right),
\end{aligned}
$$

and with the invariant under $G$, we find

$$
\begin{aligned}
\frac{4(n-1)}{n-2} \int_{M}\left|\nabla_{g} u\right|^{2} d & V_{g}+\int_{M} R_{g} u^{2} d V_{g} \\
= & k\left(\frac{4(n-1)}{n-2} \int_{M}\left|\nabla_{g} u_{1}\right|^{2} d V_{g}+\int_{M} R_{g} u_{1}^{2} d V_{g}\right) \\
& +k \sum_{i=2}^{k}\left(\frac{4(n-1)}{n-2} \int_{M}\left\langle\nabla_{g} u_{1}, \nabla_{g} u_{i}\right\rangle d V_{g}+\int_{M} R_{g} u_{1} u_{i} d V_{g}\right) .
\end{aligned}
$$

By (4.12) in [9], we have

$$
\begin{aligned}
& \frac{4(n-1)}{n-2} \int_{M}\left|\nabla u_{1}\right|^{2} d V_{g}+\int_{M} R_{g} u_{1}^{2} d V_{g} \\
& \leq Y\left(S_{+}^{n}, \partial S_{+}^{n}, g_{S_{+}^{n}}\right)\left\|u_{1}\right\|_{L^{\frac{2 n}{n-2}}(M, g)}^{2}-(n-2) A \sigma_{n-1}^{+} \epsilon_{0}^{2}+c \epsilon_{0}^{2} \epsilon^{2} \delta^{-n}+c \epsilon_{0}^{2} \delta,
\end{aligned}
$$


where $\sigma_{n-1}^{+}$is the volume of $S_{+}^{n-1}$ with respect to $g_{S_{+}^{n-1}}$. Moreover, we have

$$
\begin{aligned}
\frac{4(n-1)}{n-2} & \int_{M}\left\langle\nabla_{g} u_{1}, \nabla_{g} u_{i}\right\rangle d V_{g}+\int_{M} R_{g} u_{1} u_{i} d V_{g} \\
= & \int_{M-B_{2 \delta}\left(x_{1}\right) \cup B_{2 \delta}\left(x_{i}\right)}\left(\frac{4(n-1)}{n-2}\left\langle\nabla_{g} u_{1}, \nabla_{g} u_{i}\right\rangle+R_{g} u_{1} u_{i} d V_{g}\right) \\
& +\frac{4(n-1)}{n-2}\left(\int_{B_{2 \delta}\left(x_{1}\right)}\left\langle\nabla_{g} u_{1}, \nabla_{g} u_{i}\right\rangle d V_{g}+\int_{B_{2 \delta}\left(x_{i}\right)}\left\langle\nabla_{g} u_{1}, \nabla_{g} u_{i}\right\rangle d V_{g}\right)
\end{aligned}
$$

where we have used the fact that $g$ is flat in $B_{2 \delta}\left(x_{i}\right)$. Since $L_{g} G_{i}=0$ in $M$, and $\frac{\partial G_{i}}{\partial v_{g}}=0$ on $\partial M-\left\{x_{i}\right\}$, we infer

$$
\begin{aligned}
\int_{M-\left(B_{2 \delta}\left(x_{1}\right) \cup B_{2 \delta}\left(x_{i}\right)\right)} & \left(\frac{4(n-1)}{n-2}\left\langle\nabla_{g} u_{1}, \nabla_{g} u_{i}\right\rangle+R_{g} u_{1} u_{i}\right) d V_{g} \\
= & \epsilon_{0}^{2} \int_{M \cap \partial B_{2 \delta}\left(x_{i}\right)} \frac{\partial G_{1}}{\partial v_{i}} G_{i} d A_{g}+\epsilon_{0}^{2} \int_{M \cap \partial B_{2 \delta}\left(x_{1}\right)} \frac{\partial G_{1}}{\partial v_{1}} G_{i} d A_{g} \\
& +\epsilon_{0}^{2} \int_{\partial M-B_{2 \delta}\left(x_{1}\right)-B_{2 \delta}\left(x_{i}\right)} \frac{\partial G_{1}}{\partial v_{g}} G_{i} d A_{g} \\
= & \epsilon_{0}^{2} \int_{M \cap \partial B_{2 \delta}\left(x_{i}\right)} \frac{\partial G_{1}}{\partial v_{i}} G_{i} d A_{g}+\epsilon_{0}^{2} \int_{M \cap \partial B_{2 \delta}\left(x_{1}\right)} \frac{\partial G_{1}}{\partial v_{1}} G_{i} d A_{g}
\end{aligned}
$$

where $v_{i}$ represents the unit normal vector to $B_{2 \delta}\left(x_{i}\right)$. On $\partial B_{2 \delta}\left(x_{i}\right)$, we have $\left|\frac{\partial G_{1}}{\partial v_{i}}\right| \leq C$ and $\left|G_{i}\right| \leq C \delta^{2-n}$, which gives

$$
\epsilon_{0}^{2}\left|\int_{M \cap \partial B_{2 \delta}\left(x_{i}\right)} \frac{\partial G_{1}}{\partial v_{i}} G_{i} d A_{g}\right| \leq C \delta \epsilon_{0}^{2} .
$$

On the other hand, on $\partial B_{2 \delta}\left(x_{1}\right)$, we have $\frac{\partial G_{1}}{\partial v_{1}}=r_{1}^{1-n}+O(1)$, and $G_{i}=G_{i}\left(x_{1}\right)+O\left(r_{1}\right)$, which yields

$$
\epsilon_{0}^{2}\left|\int_{M \cap \partial B_{2 \delta}\left(x_{1}\right)} \frac{\partial G_{1}}{\partial \nu_{1}} G_{i} d A_{g}\right|=\epsilon_{0}^{2}\left|G_{i}\left(x_{1}\right)\right|+O\left(\epsilon_{0}^{2} \delta\right) .
$$

Moreover, for $i \neq j$, since $\Delta_{g} u_{j}=0$ in $B_{2 \delta}\left(x_{i}\right)$ and $\frac{\partial u_{j}}{\partial v_{g}}=0$ on $\partial M-\left\{x_{j}\right\}$, we get

$$
\begin{aligned}
\int_{B_{2 \delta}\left(x_{i}\right)}\left\langle\nabla_{g} u_{i}, \nabla_{g} u_{j}\right\rangle d V_{g} & =\int_{M \cap \partial B_{2 \delta}\left(x_{i}\right)} u_{i} \frac{\partial u_{j}}{\partial v_{i}} d A_{g}+\int_{\partial M \cap B_{2 \delta}\left(x_{i}\right)} u_{i} \frac{\partial u_{j}}{\partial v_{g}} d A_{g} \\
& =\int_{M \cap \partial B_{2 \delta}\left(x_{i}\right)} u_{i} \frac{\partial u_{j}}{\partial v_{i}} d A_{g},
\end{aligned}
$$

and with the definition of $u_{i}$, we see that

$$
\left|\frac{\partial u_{j}}{\partial v_{i}}\right| \leq C \epsilon_{0} \text { and }\left|u_{i}\right| \leq C \epsilon_{0} \delta^{2-n} \text { on } M \cap \partial B_{2 \delta}\left(x_{i}\right) .
$$

We therefore obtain

$$
\left|\int_{B_{2 \delta}\left(x_{i}\right)}\left\langle\nabla_{g} u_{i}, \nabla_{g} u_{j}\right\rangle d V_{g}\right| \leq C \delta \epsilon_{0}^{2} .
$$


Combining all these estimates, we can conclude that

$$
\begin{aligned}
\frac{4(n-1)}{n-2} \int_{M}|\nabla u|^{2} d V_{g}+\int_{M} R_{g} u^{2} d V_{g} \\
\leq k Y\left(S_{+}^{n}, \partial S_{+}^{n}, g_{S_{+}^{n}}\right)\left\|u_{1}\right\|_{L^{\frac{2 n}{n-2}}(M, g)}^{2}-k(n-2) A \sigma_{n-1}^{+} \epsilon_{0}^{2} \\
\\
\quad+c \epsilon_{0}^{2} \epsilon^{2} \delta^{-n}+c \delta \epsilon_{0}^{2}+k \epsilon_{0}^{2}\left(\sum_{i=2}^{k} G_{i}\left(x_{1}\right)\right) .
\end{aligned}
$$

It now remains to evaluate $\|u\|_{L^{\frac{2 n}{n-2}}(M, g)}^{2}$. To this end, we compute

$$
\begin{aligned}
\int_{M} u^{\frac{2 n}{n-2}} d V_{g} & \geq \sum_{i=1}^{k} \int_{B_{\delta}\left(x_{i}\right)}\left(u_{i}+\sum_{j \neq i} u_{j}\right)^{\frac{2 n}{n-2}} d V_{g} \\
& \geq \sum_{i=1}^{k} \int_{B_{\delta}\left(x_{i}\right)} u_{i}^{\frac{2 n}{n-2}} d V_{g}+\frac{2 n}{n-2} \sum_{i=1}^{k} \sum_{j \neq i} \int_{B_{\delta}\left(x_{i}\right)} u_{i}^{\frac{n+2}{n-2}} u_{j} d V_{g} \\
& \geq k \int_{B_{\delta}\left(x_{1}\right)} u_{1}^{\frac{2 n}{n-2}} d V_{g}+\frac{2 n k}{n-2} \sum_{i=2}^{k} \int_{B_{\delta}\left(x_{1}\right)} u_{1}^{\frac{n+2}{n-2}} u_{i} d V_{g}
\end{aligned}
$$

where the second inequality follows from $(a+b)^{N} \geq a^{N}+N a^{n-1} b$, and the third inequality follows from the invariance under $G$. From the definition of $u_{1}$, we have

$$
\begin{aligned}
\left\|u_{1}\right\|_{L^{\frac{2 n}{n-2}}(M, g)}^{\frac{2 n}{n-2}} & =\int_{B_{\delta}\left(x_{1}\right)} u_{1}^{\frac{2 n}{n-2}} d V_{g}+\int_{M-B_{\delta}\left(x_{1}\right)} u_{1}^{\frac{2 n}{n-2}} d V_{g} \\
& =\int_{B_{\delta}\left(x_{1}\right)} u_{1}^{\frac{2 n}{n-2}} d V_{g}+\epsilon_{0}^{\frac{2 n}{n-2}} \int_{M-B_{\delta}\left(x_{1}\right)}\left(G_{1}-\eta_{1} \alpha_{1}\right)^{\frac{2 n}{n-2}} d V_{g}
\end{aligned}
$$

and

$$
\begin{aligned}
\int_{B_{\delta}\left(x_{1}\right)} u_{1}^{\frac{2 n}{n-2}} d V_{g} & =\sigma_{n-1}^{+} \int_{0}^{\delta / \epsilon}\left(1+t^{2}\right)^{-n} t^{n-1} d t \\
& =\sigma_{n-1}^{+} \int_{0}^{\infty}\left(1+t^{2}\right)^{-n} t^{n-1} d t-\sigma_{n-1}^{+} \int_{\delta / \epsilon}^{\infty}\left(1+t^{2}\right)^{-n} t^{n-1} d t \\
& =2^{-n} \sigma_{n}^{+}+o\left(\epsilon_{0}^{2}\right)
\end{aligned}
$$

Likewise,

$$
\begin{aligned}
\int_{B_{\delta}\left(x_{1}\right)} u_{1}^{\frac{n+2}{n-2}} u_{i} d V_{g}= & \epsilon_{0} \int_{B_{\delta}\left(x_{1}\right)} u_{1}^{\frac{n+2}{n-2}} G_{i} d V_{g} \\
= & \epsilon_{0} G_{i}\left(x_{1}\right) \int_{B_{\delta}\left(x_{1}\right)} u_{1}^{\frac{n+2}{n-2}} d V_{g}+\epsilon_{0} \int_{B_{\delta}\left(x_{1}\right)} u_{1}^{\frac{n+2}{n-2}} O\left(r_{1}\right) d V_{g} \\
= & \epsilon_{0} G_{i}\left(x_{1}\right) \epsilon^{\frac{n-2}{2}} \sigma_{n-1}^{+} \int_{0}^{\delta / \epsilon}\left(1+t^{2}\right)^{-(n+2) / 2} t^{n-1} d t \\
& +\epsilon_{0} \int_{B_{\delta}\left(x_{1}\right)} u_{1}^{\frac{n+2}{n-2}} O\left(r_{1}\right) d V_{g}
\end{aligned}
$$


We can see that $\epsilon^{(n-2) / 2}$ is equivalent to $\epsilon_{0}$ from (3.2), and $\epsilon_{0} \int_{B_{\delta}\left(x_{1}\right)} u_{1}^{\frac{n+2}{n-2}} O\left(r_{1}\right) d V_{g}$ is $O\left(\epsilon_{0}^{2} \delta\right)$. Therefore, we have

$$
\begin{aligned}
E_{\frac{2 n}{n-2}}\left(u_{\delta, \epsilon}\right) \leq & k^{2 / n} Y\left(S_{+}^{n}, \partial S_{+}^{n}, g_{S_{+}^{n}}\right)-C_{0} A \epsilon_{0}^{2} \\
& +C_{1}\left(\sum_{i=2}^{k} G_{i}\left(x_{1}\right)\right)\left(1-2 n \int_{0}^{\infty}\left(1+t^{2}\right)^{-(n+2) / 2} t^{n-1} d t\right) \epsilon_{0}^{2} \\
& +O\left(\epsilon_{0}^{2} \delta\right)+o\left(\epsilon_{0}^{2}\right)
\end{aligned}
$$

where $C_{0}$ and $C_{1}$ are strictly positive constants independent of $\delta$ and $\epsilon$. Finally it remains to notice that we always have $\int_{0}^{\infty}\left(1+t^{2}\right)^{-(n+2) / 2} t^{n-1} d t=1 / n$ and if we set $C_{2}=$ $C_{1}\left(\inf _{i=2, \cdots, k} G_{i}\left(x_{1}\right)\right)$, so that $C_{1} \sum_{i=2}^{k} G_{i}\left(x_{1}\right) \geq C_{2}(k-1)$, we obtain two strictly positive constants $C_{0}$ and $C_{2}$, independent of $\delta$ and $\epsilon$, with

$$
E_{\frac{2 n}{n-2}}\left(u_{\delta, \epsilon}\right) \leq k^{2 / n} Y\left(S_{+}^{n}, \partial S_{+}^{n}, g_{S_{+}^{n}}\right)-\epsilon_{0}^{2}\left(C_{0} A+C_{2}(k-1)\right)+O\left(\epsilon_{0}^{2} \delta\right)+o\left(\epsilon_{0}^{2}\right) .
$$

In particular, we can find $\delta$ and $\epsilon$ sufficiently small so that $E_{\frac{2 n}{n-2}}\left(u_{\delta, \epsilon}\right)<k^{2 / n} Y\left(S_{+}^{n}, \partial S_{+}^{n}, g_{S_{+}^{n}}\right)$ if $C_{0} A+C_{2}(k-1)>0$. By the positive mass theorem, we have $A>0$ (cf. [9, Appendix]). Therefore we can conclude that $\mu_{\frac{2 n}{n-2}}(G)<k^{2 / n} Y\left(S_{+}^{n}, \partial S_{+}^{n}, g_{S_{+}^{n}}\right)$.

Now we suppose $M=S_{+}^{n}$. Then, since $M$ is locally conformally flat, we can follow the same argument as in the above and eventually obtain the Eq. (3.3). What makes this case different from the cases discussed earlier is the fact that, when $M=S_{+}^{n}$, we have $A=0$ (cf. [9, Appendix]). Since $C_{2}$ is a positive constant, so if $k \neq 1$, we have $\mu_{\frac{2 n}{n-2}}(G)<$ $k^{2 / n} Y\left(S_{+}^{n}, \partial S_{+}^{n}, g_{S_{+}^{n}}\right)$.

Suppose $k=$ card $O_{G}\left(x_{1}\right)=1$, i.e. $G$ admits a fixed point (denote by $x$ ). Since $x$ is a fixed point, $G$ becomes a subgroup of $I_{x}\left(S_{+}^{n}, \partial S_{+}^{n}, g\right)$. Then, by Lemma 2.5 , there exist $\tau \in C_{x}\left(S_{+}^{n}, \partial S_{+}^{n}, g_{S_{+}^{n}}\right)$ so that $G \subset \tau^{-1} I_{x}\left(S_{+}^{n}, \partial S_{+}^{n}, g_{S_{+}^{n}}\right) \tau$. Let $f, \varphi>0$ be smooth functions such that

$$
\left(\tau^{-1}\right)^{*} g_{S_{+}^{n}}=f^{\frac{4}{n-2}} g_{S_{+}^{n}}, \quad g=\varphi^{\frac{4}{n-2}} g_{S_{+}^{n}} .
$$

We set $u(x)=1 / \varphi(x) f(\tau(x))$. First, we show that $u$ is $G$-invariant. To see this, consider $\sigma \in G$ and $i \in I_{x_{1}}\left(S_{+}^{n}, \partial S_{+}^{n}, g_{S_{+}^{n}}\right)$ which satisfies $\sigma=\tau^{-1} i \tau$. Then

$$
\begin{aligned}
\sigma^{*} g & =\tau^{*} i^{*}\left(\tau^{-1}\right)^{*} g \\
& =\left(\tau^{*} i^{*}\right)\left(\left(\varphi \circ \tau^{-1}\right)^{\frac{4}{n-2}} f^{\frac{4}{n-2}} g_{S_{+}^{n}}\right) \\
& =((\varphi \circ \sigma)(f \circ i \circ \tau))^{\frac{4}{n-2}}(f \circ \tau)^{-\frac{4}{n-2}} g_{S_{+}^{n}} .
\end{aligned}
$$

But since $\sigma^{*} g=g$, we get $(\varphi \circ \tau)((f \circ \tau) \circ \sigma)=\varphi(f \circ \tau)$. This shows that the invariance of $u$ under $\sigma$, hence under $G$. Since $\varphi u=1 /(f \circ \tau)$ and $\tau^{*} g_{S_{+}^{n}}=(f \circ \tau)^{-4 /(n-2)} g_{S_{+}^{n}}$, we can easily verify that $E_{\frac{2 n}{n-2}}(u)=Y\left(S_{+}^{n}, \partial S_{+}^{n}, g_{S_{+}^{n}}\right)$. This shows that $\mu_{\frac{2 n}{n-2}}(G)=Y\left(S_{+}^{n}, \partial S_{+}^{n}, g_{S_{+}^{n}}\right)$.

Now we consider the case of $x_{1} \in M$. Even in this case, we can show that there is a metric $g^{\prime}$ that is $G$-invariant and Euclidean in the neighborhood of each point of a minimal orbit through the same method as before. Then, near $x_{i}$, the Green's function $G_{i}$ of the conformal 
Laplacian $L_{g}$ admits an expansion of the type

$$
G_{i}(x)=r_{i}^{2-n}+A+\alpha_{i}(x)
$$

where $A$ is a constant, $\alpha_{i}(x)$ is a smooth harmonic function near $x_{i}$ with $\alpha\left(x_{i}\right)=0$, and $r_{i}=d\left(x_{i}, x\right)$.

For each $x_{i}$, we now consider the function $u_{\delta, \epsilon}^{i}$ defined by

$$
u_{\delta, \epsilon}^{i}= \begin{cases}\left(\frac{\epsilon}{\epsilon^{2}+r_{i}^{2}}\right)^{(n-2) / 2}, & \text { for } x \in M \cap B_{\delta}\left(x_{i}\right) ; \\ \epsilon_{0}\left(G_{i}(x)-\eta_{i}(x) \alpha_{i}(x)\right), & \text { for } x \in M \cap\left(B_{2 \delta}\left(x_{i}\right)-B_{\delta}\left(x_{i}\right)\right) ; \\ \epsilon_{0} G_{i}(x), & \text { for } x \in M \cap\left(B_{3 \delta}\left(x_{i}\right)-B_{2 \delta}\left(x_{i}\right)\right) ; \\ \epsilon_{0} G_{i}(x) \eta_{i}^{\prime}(x), & \text { for } x \in M \cap\left(B_{4 \delta}\left(x_{i}\right)-B_{3 \delta}\left(x_{i}\right)\right) ; \\ 0, & \text { otherwise; }\end{cases}
$$

where $\delta$ is chosen small enough so that $B_{4 \delta}\left(x_{i}\right) \cap \partial M=\emptyset$, and the $\eta^{\prime}$ is a smooth cut-off function such that $0 \leq \eta_{i}^{\prime} \leq 1, \eta_{i}^{\prime}(x)=1$ if $r_{i} \leq 3 \delta, \eta_{i}^{\prime}(x)=0$ if $r_{i} \geq 4 \delta$ and $\left|\nabla_{g} \eta_{i}^{\prime}\right| \leq 2 / \delta$. We set $u_{\delta, \epsilon}=\sum_{i=1}^{k} u_{\delta, \epsilon}^{i}$ so that the function $u_{\delta, \epsilon}$ is $G$-invariant. Then we can get the following using the arguments used above:

$$
E_{\frac{2 n}{n-2}}\left(u_{\delta, \epsilon}\right) \leq k^{2 / n} Y\left(S_{+}^{n}, \partial S_{+}^{n}, g_{S_{+}^{n}}\right)-\epsilon_{0}^{2}\left(C_{0} A+C_{2}(k-1)\right)+\text { negligible terms. }
$$

Then, once again by the positive mass theorem (cf. [9, Appendix]) we obtain $\mu_{\frac{2 n}{n-2}}(G)<$ $k^{2 / n} Y\left(S_{+}^{n}, \partial S_{+}^{n}, g_{S_{+}^{n}}\right)$.

\section{The case of dimension 3, 4, and 5 for type (I)}

In this section, we prove Theorem 1.9 when $3 \leq n \leq 5$. Let $(M, g)$ be a compact $n$ dimensional Riemannian manifold with boundary $\partial M$. First assume that $\partial M$ is umbilic and that $M$ is not conformally diffeomorphic to $S_{+}^{n}$. We also suppose that $G$ has finite orbits, and we denote by $O_{G}\left(x_{1}\right)=\left\{x_{1}, \cdots, x_{k}\right\}$ a minimal orbit under $G$. In this section we only discuss the case of $x_{1} \in \partial M$. However, in the case of $x_{1} \in M$, one can obtain the desired result in the same way as $x_{1} \in \partial M$, as it was in the previous section.

Unlike the previous section, $g$ is no longer assumed to be Euclidean in the neighborhood of $x_{i}$, and as a result, some additional terms appear in the estimates. But we will see that the additional terms are always negligible in lower dimensions.

Set $\varphi_{1}$ to be the first eigenfunction for the conformal Laplacian with respect to the boundary condition $\frac{\partial \varphi}{\partial v_{g}}=0$ on $\partial M$. Then the metric $g_{1}=\varphi_{1}^{\frac{4}{n-2}} g$ has minimal boundary, and this implies that the second fundamental form vanishes on $\partial M$. Let $\left(y_{1}^{1}, \cdots, y_{n}^{1}\right)$ be geodesic normal coordinates at $x_{1} \in \partial M$.

When $n=3$, the Green's function $G_{i}$ for the conformal Laplacian with the boundary condition $\frac{\partial G}{\partial v_{g}}=0$ has the expansion near $x_{i}$ as

$$
G_{i}(y)=\left|r_{i}\right|^{-1}+A+O^{\prime \prime}\left(\left|r_{i}\right|\right)
$$

where $A$ is a constant. Here we write $f=O^{\prime}\left(r^{m}\right)$ to mean $f=O\left(r^{m}\right)$ and $\nabla_{g} f=O\left(r^{m-1}\right)$. And $O^{\prime \prime}$ is defined similarly. We define $u_{\delta, \epsilon}^{i}$, and $u_{\delta, \epsilon}$ as in the previous section. It follows 
from $[9$, section 4$]$ that

$$
8 \int_{M}\left|\nabla_{g} u_{1}\right|^{2} d V_{g}+\int_{M} R_{g} u_{1}^{2} d V_{g} \leq Y\left(S_{+}^{3}, \partial S_{+}^{3}, g_{S_{+}^{3}}\right)\left\|u_{1}\right\|_{L^{6}(M, g)}^{2}-A \sigma_{2}^{+} \epsilon_{0}^{2}+I_{3},
$$

where $I_{3}$ is the error term satisfying

$$
I_{3}=c \epsilon_{0}^{2} \epsilon^{2} \delta^{-3}+c \epsilon_{0}^{2} \delta+c \epsilon \delta+c \epsilon^{2} .
$$

As before, we can show that

$$
E_{6}\left(u_{\delta, \epsilon}\right) \leq k^{2 / 3} Y\left(S_{+}^{3}, \partial S_{+}^{3}, g_{S_{+}^{3}}\right)-\epsilon_{0}^{2}\left(C_{0} A+C_{2}(k-1)\right)+\text { negligible terms, }
$$

where $C_{0}$ and $C_{2}$ are positive constants. And the positive mass theorem (cf. [9, Appendix]) guarantees that $C_{0} A+C_{2}(k-1)>0$. So we have $\mu_{6}(G)<k^{2 / 3} Y\left(S_{+}^{3}, \partial S_{+}^{3}, g_{S_{+}^{3}}\right)$.

To prove the cases for $n=4$ and $n=5$, we need the following:

Lemma 4.1 If $n=4$ (respectively $n=5$ ), then for any $x \in \partial M$, there exists a metric $g_{x}^{\prime}$ conformal to $g$ and $I_{x}(M, \partial M, g)$-invariant which satisfies $H_{g_{x}^{\prime}}=0$ and $R c\left(g_{x}^{\prime}\right)(x)=0$ (resp. $\operatorname{Rc}\left(g_{x}^{\prime}\right)(x)=0$ and $\left.\operatorname{Sym} \nabla R c\left(g_{x}^{\prime}\right)=0\right)$.

Proof The dimension has in fact nothing to do here. We will first show that given a compact Riemannian manifold $(M, g)$ with boundary $\partial M$ and $x$ a point of $\partial M$, we can always find a $I_{x}(M, \partial M, g)$-invariant metric $g_{x}^{\prime}$ conformal to $g$, which satisfies $H_{g_{x}^{\prime}}(x)=0$ and $R c\left(g_{x}^{\prime}\right)(x)=0$.

We consider $\Omega$ an open neighborhood of 0 in $T_{x} M$ so that $\left.\exp _{x}\right|_{\Omega}$ is a diffeomorphism. If $\sigma$ is an isometry of $I_{x}(M, \partial M, g), d \sigma(x) \in O\left(T_{x} M, g(x)\right)$ and

$$
d \sigma(x)=\exp _{x}^{-1} \circ \sigma \circ \exp _{x} \text { on } \Omega .
$$

The mapping $\psi: I_{x}(M, \partial M, g) \rightarrow O\left(T_{x} M, g(x)\right)$ defined by $\psi(\sigma)=d \sigma(x)$ becomes an injective morphism of groups, so that $I_{x}(M, \partial M, g)$ identifies with a subgroup of $O\left(T_{x} M, g(x)\right)$. We now consider $R c(g)(x)$, which is a symmetrical bilinear form over $T_{x}(X)$. Let $\left(e_{1}, \cdots, e_{n}\right)$ be an orthonormal basis of $T_{x} M$ which diagonalizes $R c(g)(x)$, and $E_{i}, i=1, \cdots, n$ the associated eigenspace. Then the invariance of $R c(g)(x)$ under $I_{x}(M, \partial M, g)$ imposes the invariance of $E_{i}$ under the same group. Let $r_{i}$ be the distance from the origin of $T_{x} M$ measured on $E_{i}$ and $a^{1}, \cdots, a^{n}$ real numbers. Then we consider $\tilde{f}: T_{x} M \rightarrow \mathbb{R}$ defined by $\tilde{f}\left(e_{i}\right)=a^{i} r_{i}^{2}$. The function $\tilde{f}$ is clearly $I_{x}(M, \partial M, g)$-invariant so that the function $f: \exp _{x}(\Omega) \rightarrow \mathbb{R}$ defined by $f=\tilde{f} \circ \exp _{x}^{-1}$ also becomes $I_{x}(M, \partial M, g)$ invariant. We now show that it is possible to choose the $a^{i}$ (in fact in a unique way) so that $f$ satisfies $R c\left(e^{f} g\right)(x)=0$.

One can easily see that $f(x)=0$ and $\nabla_{g} f(x)=0$, so we have

$$
R c\left(e^{f} g\right)(x)=R c(g)(x)-\frac{n-2}{2}\left(\nabla_{g}^{2} f\right)(x)+\frac{1}{2} \Delta_{g} f(x) g(x)
$$

and in the normal map $\left(\exp _{x}(\Omega), \exp _{x}^{-1}\right)$ associated with $\left(e_{1}, \cdots, e_{n}\right)$, we can see that $R c\left(e^{f} g\right)(x)=0$ is equivalent to

$$
\left(\begin{array}{lll}
\lambda_{1} & & \\
& \ddots & \\
& & \lambda_{n}
\end{array}\right)-(n-2)\left(\begin{array}{ccc}
a^{1} & & \\
& \ddots & \\
& & a^{n}
\end{array}\right)-\left(\sum_{i=1}^{n} a^{i}\right) I d=0 .
$$

It is clear that this system always admits a solution (which is unique). Now we set $g_{x}^{\prime}=$ $\eta\left(e^{f} g\right)+(1-\eta) g$. This proves the first part of Lemma 4.1 . 
For the second part, it remains to show that we can also have $\operatorname{Sym} \nabla R c(x)=0$. Although it is more technical, basically we can adopt the same method as in the first part. Starting from the metric $g_{x}^{\prime}$ which has just been obtained, it will be a question of constructing a homogeneous polynomial $\tilde{f}$ of third degree so that

$$
\operatorname{Sym} \nabla R c\left(e^{f} g_{x}^{\prime}\right)(x)=0 .
$$

In order not to overload the drafting, we are leaving this point aside.

Now we consider the metric

$$
g^{\prime}=\sum_{i=1}^{k}\left(\sigma_{i}^{-1}\right)^{*}\left(\eta g_{x_{1}}\right)+\left[\prod_{i=1}^{k}\left(1-\eta \circ \sigma_{i}^{-1}\right)\right] g .
$$

Then we can see that $g^{\prime}$ is conformal to $g, G$-invariant, and satisfies $R c\left(g^{\prime}\right)=0$ (resp. $R c\left(g^{\prime}\right)=0$ and $\left.\nabla R c\left(g^{\prime}\right)=0\right)$ and $H\left(g^{\prime}\right)=0$ at any point of a minimal orbit under $G$.

Let us point out that, by the same reasoning of recurrence of this type, it is possible to prove the existence of conformal normal $G$-invariant coordinates. More precisely, we have the following:

Lemma 4.2 Given a compact Riemanian manifold $(M, g)$ with boundary $\partial M, x$ a point of $\partial M$, and a poistive integer $m$, there exists a $I_{x}(M, \partial M, g)$-invariant metric $g_{x}^{\prime}$ conformal to $g$ such that, in a normal geodesic map in $x$,

$$
\operatorname{det} g_{x}^{\prime}=1+O\left(r^{m}\right)
$$

where represents the distance to point $x$. In particular, if $G$ is a subgroup of $I(M, \partial M, g)$ and if $x \in \partial M$ has a finite orbit under $G$, then for any positive integer $m$, we can associate a $G$-invariant metric $g^{\prime}$ conformal to $g$ and so that

$$
\operatorname{det} g^{\prime}=1+O\left(r^{m}\right)
$$

in a normal geodesic coordinates at any point in the orbit of $x$ under $G$.

By Lemma 4.1, when $n=4$, the Green function's $G_{i}$ for the conformal Laplacian with boundary conditions $\frac{\partial G_{i}}{\partial v_{g}}=0$ has the expansion for $x_{i}$ small as

$$
G_{i}(x)=|x|^{-2}+A+O^{\prime \prime}(|x| \log |x|)
$$

where $A$ is a constant. We define $u_{\delta, \epsilon}^{i}$ and $u_{\delta, \epsilon}$ as in section 4. It follows from [9, section 4] that

$$
6 \int_{M}\left|\nabla u_{1}\right|^{2} d V_{g}+\int_{M} R_{g} u_{1}^{2} d V_{g} \leq Y\left(S_{+}^{4}, \partial S_{+}^{4}, g_{S_{+}^{4}}\right)\left\|u_{1}\right\|_{L^{4}(M, g)}^{2}-2 A \sigma_{3}^{+} \epsilon_{0}^{2}+I_{4}
$$

where $I_{4}$ is the error term satisfying

$$
I_{4}=c \epsilon_{0}^{2} \epsilon^{2} \delta^{-4}+c \epsilon_{0}^{2} \delta+c \epsilon^{2} \delta+c \epsilon^{3}+c \epsilon^{3} \log \left(\delta \epsilon^{-1}\right)+c \delta \log \delta^{-1} \epsilon_{0}^{2} .
$$

Then we can show that

$$
E_{4}\left(u_{\delta, \epsilon}\right) \leq k^{1 / 2} Y\left(S_{+}^{4}, \partial S_{+}^{4}, g_{S_{+}^{4}}\right)-\epsilon_{0}^{2}\left(C_{0} A+C_{2}(k-1)\right)+\text { negligible terms }
$$

where $C_{0}$ and $C_{2}$ are positive constants. And the positive mass theorem guarantees that $C_{0} A+C_{2}(k-1)>0$. So we have $\mu_{4}(G)<k^{1 / 2} Y\left(S_{+}^{4}, \partial S_{+}^{4}, g_{S_{+}^{4}}\right)$. 
Suppose $n=5$. Let $\left(y_{1}^{i}, \cdots, y_{n}^{i}\right)$ denote a normal rectangular coordinate system centered at $x_{i}$. Let $r_{i}=\left|y^{i}\right|, \zeta=y_{i} /\left|y^{i}\right|$. Then the metric $g$ of $M$ can be locally written as

$$
g=d r_{i}^{2}+r_{i}^{2} h_{r_{i}}
$$

where $h_{r_{i}}$ is a metric on $S_{+}^{n-1}$ with $h_{0}$ being the standard metric on $S_{+}^{n-1}$. Given $\rho>0$ with $\rho$ small, let $\xi\left(r_{i}\right)$ be a smooth nonincreasing function satisfying $\xi\left(r_{i}\right)=1$ for $r_{i} \leq \rho$, and $\xi\left(r_{i}\right)=0$ for $r_{i} \geq 2 \rho$; let $\left|\xi^{\prime}\left(r_{i}\right)\right| \leq c \rho^{-1}$ and let $\left|\xi^{\prime \prime}\left(r_{i}\right)\right| \leq c \rho^{-2}$ for all $r_{i}>0$. We define the metric ${ }^{\rho} g$ on $M$ by setting ${ }^{\rho} g=0$ on $M-B_{2 \rho}^{+}$and

$$
{ }^{\rho} g=d r_{i}^{2}+r_{i}^{2}\left(\xi\left(r_{i}\right) h_{0}+\left(1-\xi\left(r_{i}\right)\right) h_{r_{i}}\right) \text { whenever } r_{i} \leq 2 \rho .
$$

Thus ${ }^{\rho} g$ is Euclidean in $B_{\rho}^{+}$and coincides with $g$ outside $B_{2 \rho}^{+}$. It is easy to check that the curvature tensor of ${ }^{\rho} \mathrm{g}$ is bounded, independent of $\rho$. It follows from [8, Theorem 7.1] that the boundary $\partial M$ is totally geodesic with respect to the metric ${ }^{\rho} g$.

Let $L_{\rho}$ and $B_{\rho}$ denote the following linear operators taken in terms of ${ }^{\rho} g$ :

$$
L_{\rho}=-\frac{4(n-1)}{n-2} \Delta_{\rho}+R_{\rho}, \quad B_{\rho}=\frac{\partial}{\partial v_{\rho}} .
$$

Let $\lambda_{\rho}$ denote the lowest eigenvalue of $L_{\rho}$, and $\lambda$ the lowest eigenvalue of $L$. Then since $g$ is conformally related to a metric with positive scalar curvature, where the boundary is minimal, we have $\lambda>0$. Let $G_{i}$ be the multiple of the Green's function of $(L, B)$ with pole at $x_{i}$ and normalize so that $\lim _{\left|y^{i}\right| \rightarrow 0}\left|y^{i}\right|^{3} G_{i}\left(y^{i}\right)=1$. Note that ${ }^{\rho} g$ converges in the $C^{1}$-norm to $g$ as $\rho \searrow 0$ and $R_{\rho}$ is uniformly bounded. Thus, for $\rho$ sufficiently small, $\lambda_{\rho}>0$. Hence we can consider $G_{i, \rho}$ to be the positive Green's function $\left(L_{\rho}, B_{\rho}\right)$. Normalize the function $G_{i, \rho}$ such that $\lim _{\left|y^{i}\right| \rightarrow 0}\left|y^{i}\right|^{3} G_{i, \rho}=1$. Consider the double of $M, \tilde{M}=M \cup \partial M \cup M$, equipped with the metric induced from $M$. Then we can show that the function $G_{i, \rho}$ converges to $G_{i}$ as $\rho \rightarrow 0$ in the $C^{2}$-norm on compact subsets of $\bar{M}-\left\{x_{i}\right\}$ by applying Lemma 1 in [17]. Since the metric ${ }^{\rho} g$ are Euclidean in $B_{\rho}\left(x_{i}\right)$, the function $G_{i, \rho}$ is harmonic in $B_{\rho}$ and hance has an expansion for $\left|y^{i}\right|$ small,

$$
G_{i, \rho}\left(y^{i}\right)=\left|y^{i}\right|^{-3}+A_{\rho}+O\left(\left|y^{i}\right|\right), \quad A_{\rho} \geq 0 .
$$

We define $u_{\delta, \epsilon}^{i}$, and $u_{\delta, \epsilon}$ as in the previous section. It follows from (2.8) in [17] that

$$
\begin{aligned}
\frac{16}{3} \int_{M}\left|\nabla u_{1}\right|^{2} d V_{g}+\int_{M} R_{g} u_{1}^{2} d V_{g} \leq & Y\left(S_{+}^{5}, \partial S_{+}^{5}, g_{\left.\left.S_{+}^{5}\right)\left\|u_{1}\right\|_{L^{\frac{10}{3}}}^{2}-3, g\right)}-3 \sigma_{4}^{+} A_{\rho} \epsilon_{0}^{2}\right. \\
& +c \rho_{0}^{-5} \epsilon_{0}^{\frac{8}{3}}+c \rho_{0} \epsilon_{0}^{2}+c_{1} \rho^{\frac{1}{2}} \epsilon_{0}^{2},
\end{aligned}
$$

where $c$ depends on $\rho$ but $c_{1}$ does not. Then we can show that

$$
E_{\frac{10}{3}}\left(u_{\delta, \epsilon}\right) \leq k^{2 / 5} Y\left(S_{+}^{5}, \partial S_{+}^{5}, g_{S_{+}^{5}}\right)-\epsilon_{0}^{2}\left(C_{0} A_{\rho}+C_{2}(k-1)+\right.\text { negligible terms }
$$

where $C_{0}$ and $C_{2}$ are positive constants. Since we assumed that $M$ is not conformally equivalent to $S_{+}^{5}$, it follows from [9, Section4] that

$$
\liminf _{\rho \rightarrow 0} A_{\rho}>0 \text {. }
$$

So, fixing $\rho$ small, then fixing $\rho_{0}$, and finally choosing $\epsilon_{0}$ sufficiently small, we prove $\mu_{\frac{10}{3}}(G)<k^{2 / 5} Y\left(S_{+}^{5}, \partial S_{+}^{5}, g_{S_{+}^{5}}\right)$. 


\section{Notations and preliminary results for type (II)}

Let $(M, \partial M, g)$ be a compact $n$-dimensional Riemannian manifold with smooth boundary $\partial M$. By conformal change of $g$, we may assume that $R_{g}=0$ in $M$ (see Proposition 1.4 in [8]). It is known that if $Q(M, \partial M, g) \leq 0$, then the Equivariant Yamabe problem with boundary (II) is always solvable; for one can seek the solution to the subcritical case, pass to the limit and get the solution to the critical case, as done by Trudinger for the classical Yamabe problem. More precisely, for all $2<q \leq \frac{2(n-1)}{n-2}$, there exists $u_{q}>0$ such that $\left.u_{q}\right|_{\partial M}$ is $G$-invariant and satisfies

$$
\Delta_{g} u_{q}=0 \text { in } M, \quad \frac{2(n-1)}{n-2} \frac{\partial u_{q}}{\partial v_{g}}+H_{g} u_{q}=\frac{\mu_{q}(G)}{2} u_{q}^{q-1} \text { on } \partial M .
$$

where $\mu_{q}(G)$ is defined below. By the assumption $Y(M, \partial M, g) \leq 0, u_{q}$ converges to $u$ as $q \rightarrow \frac{2(n-1)}{n-2}$, where $u$ is a solution to the Equivariant Yamabe problem with boundary (II).

Hence, we assume that $Q(M, \partial M, g)>0$. By a conformal change of $g$, we may assume that the scalar curvature of $g$ vanishes in $M$ (see Proposition 1.4 in [8]), i.e.

$$
R_{g}=0 \text { in } M \text { and } H_{g}>0 \text { on } \partial M .
$$

Let

$$
H^{\frac{1}{2}}(\partial M)=\left\{\left.u\right|_{\partial M}: u \in H_{1}^{2}(M), \Delta_{g} u=0 \text { in } M\right\} .
$$

Note that $H^{\frac{1}{2}}(\partial M) \subseteq L^{\frac{2(n-1)}{n-2}}(\partial M)$ by the Sobolev trace embedding (0.4) in [13].

For $2 \leq q \leq \frac{2(n-1)}{n-2}$, we define

$$
Q_{q}(u)=\frac{\int_{M} \frac{4(n-1)}{n-2}\left|\nabla_{g} u\right|^{2} d V_{g}+2 \int_{\partial M} H_{g} u^{2} d A_{g}}{\left(\int_{\partial M} u^{q} d A_{g}\right)^{\frac{2}{q}}},
$$

where $0 \not \equiv u \in H_{1}^{2}(M)$. Note that $Q_{\frac{2(n-1)}{n-2}}(u)=Q(u)$ defined in (1.7) thanks to (5.1). For $G \subset I\left(\partial M,\left.g\right|_{\partial M}\right)$ and $2 \leq q \leq \frac{2(n-1)}{n-2}$, we define

$$
\mu_{q}(G)=\inf \left\{Q_{q}(u): 0 \not \equiv u \in H^{\frac{1}{2}}(\partial M) \text { and }\left.u\right|_{\partial M} \text { is } G \text {-invariant }\right\}
$$

It is well-defined, since $H^{\frac{1}{2}}(\partial M) \subseteq L^{q}(\partial M)$ for any $2 \leq q \leq \frac{2(n-1)}{n-2}$.

Lemma 5.1 For all $2 \leq q<\frac{2(n-1)}{n-2}$ and all $G \subseteq I\left(\partial M,\left.g\right|_{\partial M}\right), \mu_{q}(G)$ is attained. Thus there exists $0<u_{q} \in C^{\infty}(\bar{M})$ such that $\left.u_{q}\right|_{\partial M}$ is $G$-invariant and

$$
\Delta_{g} u_{q}=0 \text { in } M, \quad \frac{2(n-1)}{n-2} \frac{\partial u_{q}}{\partial v_{g}}+H_{g} u_{q}=\frac{\mu_{q}(G)}{2} u_{q}^{q-1} \text { on } \partial M, \quad \int_{\partial M} u_{q}^{q} d A_{g}=1 \text {. }
$$

Proof Consider a sequence $\left\{u_{i}\right\}$ of positive functions in $H_{1}^{2}(M)$ such that $\left.u_{i}\right|_{\partial M}$ is $G$ invariant, satisfies $\Delta_{g} u_{i}=0$ in $M$ and

$$
\lim _{i \rightarrow \infty} Q_{q}\left(u_{i}\right)=\mu_{q}(G) \text { and } \int_{M} u_{i}^{q} d A_{g}=1 .
$$


Since the embedding $H^{\frac{1}{2}}(\partial M) \hookrightarrow L^{q}(\partial M)$ is compact for all $2 \leq q<\frac{2(n-1)}{n-2}$, by passing to a subsequence, we can suppose

$$
\begin{aligned}
& \lim _{i \rightarrow \infty} u_{i}=u_{q} \text { strongly in } L^{2}(\partial M) \cap L^{q}(\partial M), \\
& \lim _{i \rightarrow \infty} u_{i}=u_{q} \text { weakly in } H^{\frac{1}{2}}(\partial M), \text { and } \\
& \lim _{i \rightarrow \infty} u_{i}=u_{q} \text { almost everywhere. }
\end{aligned}
$$

It follows from (5.5) that $u_{q}$ is positive almost everywhere. Since $\left.u_{i}\right|_{\partial M}$ is $G$-invariant, we have $u_{i}(\gamma x)=u_{i}(x)$ for all $\gamma \in G$ and all $x \in \partial M$, which together with (5.5) implies that $u_{q}(\gamma x)=\lim _{i \rightarrow \infty} u_{i}(\gamma x)=\lim _{i \rightarrow \infty} u_{i}(x)=u_{q}(x)$, i.e. $\left.u_{q}\right|_{\partial M}$ is $G$-invariant. It also follows from (5.2) and (5.3) that

$$
1=\lim _{i \rightarrow \infty} \int_{\partial M} u_{i}^{q} d A_{g}=\int_{\partial M} u_{q}^{q} d A_{g} \text { and } \lim _{i \rightarrow \infty} \int_{\partial M} H_{g} u_{i}^{2} d A_{g}=\int_{\partial M} H_{g} u_{q}^{2} d A_{g} .
$$

Note that

$$
\begin{aligned}
0 & \leq \int_{M}\left|\nabla_{g} u_{i}-\nabla_{g} u_{q}\right|^{2} d V_{g} \\
& =\int_{M}\left|\nabla_{g} u_{i}\right|^{2} d V_{g}+\int_{M}\left|\nabla_{g} u_{q}\right|^{2} d V_{g}-2 \int_{M}\left\langle\nabla_{g} u_{i}, \nabla u_{q}\right\rangle d V_{g} .
\end{aligned}
$$

By (5.4), we have

$$
\lim _{i \rightarrow \infty} \int_{M}\left\langle\nabla_{g} u_{i}, \nabla u_{q}\right\rangle d V_{g}=\int_{M}\left|\nabla u_{q}\right|^{2} d V_{g}
$$

This together with (5.7), we get

$$
\lim _{i \rightarrow \infty} \int_{M}\left|\nabla_{g} u_{i}\right|^{2} d V_{g} \geq \int_{M}\left|\nabla_{g} u_{q}\right|^{2} d V_{g}
$$

By (5.1), (5.2), (5.6) and (5.8), we have

$$
\begin{aligned}
Q_{q}\left(u_{q}\right) & =\int_{M} \frac{4(n-1)}{n-2}\left|\nabla_{g} u_{q}\right|^{2} d V_{g}+\int_{\partial M} H_{g} u_{q}^{2} d A_{g} \\
& \leq \lim _{i \rightarrow \infty}\left(\int_{M} \frac{4(n-1)}{n-2}\left|\nabla_{g} u_{i}\right|^{2} d V_{g}+\int_{\partial M} H_{g} u_{i}^{2} d A_{g}\right) \\
& =\lim _{i \rightarrow \infty} Q_{q}\left(u_{i}\right)=\mu_{q}(G) .
\end{aligned}
$$

This implies that $u_{q}$ realizes the minimum of $Q_{q}(u)$ on the space of $u \in H^{\frac{1}{2}}(\partial M)$ such that $\left.v\right|_{\partial M}$ is $G$-invariant. The Euler-Lagrange equation then implies that

$$
\int_{M} \frac{4(n-1)}{n-2}\left\langle\nabla_{g} u_{q}, \nabla_{g} v\right\rangle d V_{g}+2 \int_{\partial M} H_{g} u_{q} v d A_{g}=\frac{\mu_{q}(G)}{2} \int_{\partial M} u_{q}^{q-1} v d A_{g}
$$

for all $v \in H^{\frac{1}{2}}(\partial M)$ such that $\left.v\right|_{\partial M}$ is $G$-invariant. It also follows from (5.4) that

$$
\Delta_{g} u_{q}=\lim _{i \rightarrow \infty} \Delta_{g} u_{i}=0 \text { in } M .
$$


Let $B_{g}$ be the boundary operator of $g$ :

$$
B_{g}(u)=\frac{2(n-1)}{n-2} \frac{\partial u}{\partial v_{g}}+H_{g} u \text { for } u \in H^{\frac{1}{2}}(M) .
$$

Since $H_{g}>0$ by (5.1), the boundary operator $B_{g}$ defined in $H^{\frac{1}{2}}(M)$ is invertible. Hence, let $w \in H^{\frac{1}{2}}(M)$ be the unique solution of the equation

$$
B_{g}(w)=\frac{\mu_{q}(G)}{2} u_{q}^{q-1}
$$

Since $\left.u_{q}\right|_{\partial M}$ is $G$-invariant, the uniqueness of $w$ shows that $\left.w\right|_{\partial M}$ is also $G$-invariant. On the other hand, it follows from (5.9) and (5.11) that

$$
\int_{M} \frac{4(n-1)}{n-2}\left\langle\nabla_{g}\left(u_{q}-w\right), \nabla_{g} v\right\rangle d V_{g}+2 \int_{\partial M} H_{g}\left(u_{q}-w\right) v d A_{g}=0
$$

for all functions $v \in H^{\frac{1}{2}}(M)$ such that $\left.v\right|_{\partial M}$ is $G$-invariant. Since $\left.\left(u_{q}-w\right)\right|_{\partial M}$ is $G$-invariant, we have

$$
\begin{aligned}
0 & =\int_{M} \frac{4(n-1)}{n-2}\left|\nabla_{g}\left(u_{q}-w\right)\right|^{2} d V_{g}+2 \int_{\partial M} H_{g}\left(u_{q}-w\right)^{2} d A_{g} \\
& =2 \int_{\partial M}\left(u_{q}-w\right) B_{g}\left(u_{q}-w\right) d A_{g}
\end{aligned}
$$

where we have used (5.10) and integration by parts. Since $B_{g}$ is invertible, $u_{q}=w$, i.e. $u_{q}$ is a weak solution of

$$
B_{g}\left(u_{q}\right)=\frac{\mu_{q}(G)}{2} u_{q}^{q-1}
$$

A regularity theorem of Cherrier [6] asserts that $u_{q} \in C^{\infty}(\bar{M})$. This proves the assertion.

Lemma 5.2 If a subsequence of the sequence $\left\{u_{q}\right\}_{2 \leq q<\frac{2(n-1)}{n-2}}$ converges strongly in $L^{2}(\partial M)$ to a function $0 \not \equiv u \in H^{\frac{1}{2}}(M)$, then $u$ realizes the minimum of $Q_{\frac{2(n-1)}{n-2}}$ in the space of functions in $H^{\frac{1}{2}}(M)$ which are $G$-invariant. In particular,

(i) $u$ is a positive smooth $G$-invariant function in $M$,

(ii) $\Delta_{g} u=0$ in $M$ and $\frac{2(n-1)}{n-2} \frac{\partial u}{\partial v_{g}}+H_{g} u=\frac{1}{2} \mu_{\frac{2(n-2)}{n-2}}(G) u^{\frac{n}{n-2}}$ on $\partial M$, and

(iii) $\int_{\partial M} u^{\frac{2(n-1)}{n-2}} d A_{g}=1$.

Proof First of all, we note that

$$
\limsup _{q \rightarrow \frac{2(n-1)}{n-2}} \mu_{q}(G) \leq \mu_{\frac{2(n-1)}{n-2}}(G) .
$$

To see this, it suffices to consider $0<v \in C^{\infty}(\bar{M})$ such that $\left.v\right|_{\partial M}$ is $G$-invariant and satisfies $Q_{\frac{2(n-1)}{n-2}}(v) \leq \mu_{\frac{2(n-1)}{n-2}}(G)+\epsilon$. As $\mu_{q}(G) \leq Q_{q}(v)$ with $\lim _{q \rightarrow \frac{2(n-1)}{n-2}} Q_{q}(v)=Q_{\frac{2(n-1)}{n-2}}(v)$, we get

$$
\lim _{q \rightarrow \frac{2(n-1)}{n-2}} \mu_{q}(G) \leq \lim _{q \rightarrow \frac{2(n-1)}{n-2}} Q_{q}(v)=Q_{\frac{2(n-1)}{n-2}}(v) \leq \mu_{\frac{2(n-1)}{n-2}}(G)+\epsilon .
$$


Without loss of generality, we suppose now that the subsequence $\left\{u_{q}\right\}$ satisfies $\lim u_{q}=u$ strongly in $L^{2}(\partial M) \cap L^{\frac{2(n-1)}{n-2}}(\partial M)$, weakly in $H^{\frac{1}{2}}(M)$ and almost everywhere with

$$
\mu=\lim \mu_{q}(G) \leq \frac{\mu_{\frac{2(n-1)}{n-2}}(G) .}{.}
$$

By reasoning as in the proof of Lemma 5.1, we see that $0<u \in C^{\infty}(\bar{M})$ such that $\left.u\right|_{\partial M}$ is $G$-invariant and

$$
\Delta_{g} u=0 \text { in } M \text { and } \frac{2(n-1)}{n-2} \frac{\partial u}{\partial v_{g}}+H_{g} u=\frac{\mu}{2} u^{\frac{n}{n-2}} \text { on } \partial M .
$$

It remains to show that $\mu=\mu_{\frac{2(n-1)}{n-2}}(G)$ and $\int_{\partial M} u^{\frac{2(n-1)}{n-2}} d A_{g}=1$. We find

$$
\begin{aligned}
\int_{\partial M} u^{\frac{2(n-1)}{n-2}} d A_{g} & =\lim _{q \rightarrow \frac{2(n-1)}{n-2}} \int_{\partial M} u_{q}^{\frac{n}{n-2}} u d A_{g} \\
& \leq \lim _{q \rightarrow \frac{2(n-1)}{n-2}}\left(\int_{\partial M} u_{q}^{q} d A_{g}\right)^{\frac{n}{(n-2) q}}\left(\int_{\partial M} u^{\frac{(n-2) q}{(n-2) q-n}} d A_{g}\right)^{1-\frac{n}{(n-2) q}} \\
& \leq\left(\int_{\partial M} u^{\frac{2(n-1)}{n-2}} d A_{g}\right)^{\frac{n-2}{n-1}}
\end{aligned}
$$

which implies that

$$
\int_{\partial M} u^{\frac{2(n-1)}{n-2}} d A_{g} \leq 1
$$

Multiplying the second equation in (5.13) by $u$, integrating over $\partial M$ and using the first equation in (5.13), we get

$$
\mu_{\frac{2(n-1)}{n-2}}(G) \leq Q_{\frac{2(n-1)}{n-2}}(u)=\mu\left(\int_{\partial M} u^{\frac{2(n-1)}{n-2}} d A_{g}\right)^{\frac{1}{n-1}} \leq \mu,
$$

where the last inequality follows from (5.14). Combining (5.12), (5.14) and (5.15), we can conclude that $\mu=\mu_{\frac{2(n-1)}{n-2}}(G)$ and $\int_{\partial M} u^{\frac{2(n-1)}{n-2}} d A_{g}=1$, as required.

It now remains to study the case when Lemma 5.2 does not happen. So for any subsequence of $\left\{u_{q}\right\}_{2 \leq q<\frac{2(n-1)}{n-2}}$, which converges in $L^{p}(\partial M)$ with $2 \leq p \leq \frac{2(n-1)}{n-2}$, converges to zero. This phenomena occurs when $\left\{u_{q}\right\}$ has concentration points.

For $r>0$ and $x \in \partial M$, set $B(x, r)=\left\{y \in \bar{M}: d_{g}(x, y)<r\right\}$ and $\partial^{\prime} B(x, r)=$ $\partial B(x, r) \cap \partial M$. Here, $d_{g}(x, y)$ is the distance between $x$ and $y$ in $\bar{M}$ with respect to the Riemannian metric $g$.

Lemma 5.3 In the situation described above, there exists a finite number of points $\left\{x_{1}, \cdots, x_{k}\right\}$ in $\partial M$ for which

$$
\inf _{G} Q(u)\left(\limsup _{q \rightarrow \frac{2(n-1)}{n-2}} \int_{\partial^{\prime} B\left(x_{i}, \delta\right)} u_{q}^{q} d A_{g}\right)^{\frac{1}{n-1}} \geq Q\left(D^{n}, \partial D^{n}, g_{D^{n}}\right)
$$

for all $\delta>0$ and for each $i=1,2 \ldots, k$. Furthermore, for all $p \in \mathbb{N}$, the sequence $\left\{u_{q}\right\}_{2 \leq q<\frac{2(n-1)}{n-2}}$ converges in $C^{p}(\bar{M})$ to zero on any compact set of $\bar{M}-\left\{x_{1}, \cdots, x_{k}\right\}$. 
Proof It follows from Lemma 4.1 in [21].

Proof of Theorem 1.11 By the assumption in Theorem 1.11, $\mu_{\frac{2(n-1)}{n-2}}(G)$ is strictly smaller than $Q\left(D^{n}, \partial D^{n}, g_{D^{n}}\right)\left(\inf _{x \in \partial M} \operatorname{card} O_{G}(x)\right)^{\frac{1}{n-1}}$ and it is about to show that the situation described above cannot occur (so that Lemma 5.2 applies).

Suppose that for any subsequence of $\left\{u_{q}\right\}_{2 \leq q \leq \frac{2(n-1)}{n-2}}$ converges to zero. Then by Lemma 5.3 there exists $x_{1}, \cdots, x_{k} \in \partial M$ such that $\left\{u_{q}\right\}_{2 \leq q<\frac{2(n-1)}{n-2}}$ converges to zero in $\bar{M}-\left\{x_{1}, \cdots, x_{k}\right\}$ and for each $i=1, \ldots, k$ and for all $\delta>0$

$$
\mu_{\frac{2(n-1)}{n-2}}(G)\left(\limsup _{q \rightarrow \frac{2(n-1)}{n-2}} \int_{\partial^{\prime} B\left(x_{i}, \delta\right)} u_{q}^{q} d A_{g}\right)^{\frac{1}{n-1}} \geq Q\left(D^{n}, \partial D^{n}, g_{D^{n}}\right) .
$$

We now use the property that $\left.u_{q}\right|_{\partial M}$ is invariant under $G$ and

$$
\int_{\partial M} u_{q}^{q} d A_{g}=1
$$

Two cases arise. In the first case, the orbit of $x_{i}$ in $G$ is finite, i.e. card $O_{G}\left(x_{i}\right)$ is finite. It follows from (5.17) that we can choose $\delta>0$ small enough to get $\int_{\partial^{\prime} B\left(x_{i}, \delta\right)} u_{q}^{q} d A_{g} \leq$ $\left(\operatorname{card} O_{G}\left(x_{i}\right)\right)^{-1}$, which gives

$$
\limsup _{q \rightarrow \frac{2(n-1)}{n-2}} \int_{\partial^{\prime} B\left(x_{i}, \delta\right)} u_{q}^{q} d A_{g} \leq\left(\operatorname{card} O_{G}\left(x_{i}\right)\right)^{-1} .
$$

In the second case, card $O_{G}\left(x_{i}\right)=\infty$. It is then easy to see that, for all $\epsilon>0$, there exists $0<\delta \ll 1$ with $\limsup _{q \rightarrow \frac{2(n-1)}{n-2}} \int_{\partial^{\prime} B\left(x_{i}, \delta\right)} u_{q}^{q} d A_{g} \leq \epsilon$. But if $\epsilon$ is sufficiently small, this equality contradicts (5.16).

Therefore, for all $i=1, \ldots, k$, the orbit of $x_{i}$ in $G$ is finite and

$$
\mu_{\frac{2(n-1)}{n-2}}(G) \geq Q\left(D^{n}, \partial D^{n}, g_{D^{n}}\right)\left(\operatorname{card} O_{G}\left(x_{i}\right)\right)^{\frac{1}{n-1}}
$$

by (5.16) and (5.18). The hypothesis that the strict inequality holds in (1.9), i.e.

$$
\mu_{\frac{2(n-1)}{n-2}}(G)<Q\left(D^{n}, \partial D^{n}, g_{D^{n}}\right)\left(\inf _{x \in M} \operatorname{card} O_{G}(x)\right)^{\frac{1}{n-1}}
$$

contradicts to (5.19). Therefore, $x_{i}$ in fact does not exist, and $\left\{u_{q}\right\}_{2 \leq q<\frac{2(n-1)}{n-2}}$ satisfies the conditions in Lemma 5.2. This proves Theorem 1.11.

We remark that we just showed that if the orbit of any point in $G$ is infinite, then $\mu_{\frac{2(n-1)}{n-2}}(G)$ is attained.

Proof of Theorem 1.10 Suppose $G \subset I\left(\partial M,\left.g\right|_{\partial M}\right)$ possesses a finite orbit. Let $x_{1}$ be the point of minimal orbit in $G$, i.e. card $O_{G}\left(x_{1}\right)=\inf _{x \in \partial M}$ card $O_{G}(x)$. By conformally changing $g$, we may assume that the scalar curvature of $g$ vanishes and the mean curvature of $g$ at $x_{1}$ is 
zero, i.e. $R_{g}=0$ in $M$ and $H_{g}\left(x_{1}\right)=0$. To see this, suppose that $f \in C^{\infty}(\partial M)$ such that $f$ is $G$-invariant and is strictly positive in at least one point of $\partial M$ which satisfies $f\left(x_{1}\right)=0$. Since the orbit of $x_{1}$ in $G$ is finite, it is always possible to construct such a function $f$. Let $0<u \in C^{\infty}(\bar{M})$ be the unique solution of

$$
\Delta_{g} u=0 \text { in } M \text { and } B_{g}(u)=f u^{q} \text { on } \partial M
$$

where $2 \leq q<\frac{2(n-1)}{n-2}$. Since $f$ is $G$-invariant, $\left.u\right|_{\partial M}$ is also $G$-invariant. Then the conformal metric $\widetilde{g}=u^{\frac{4}{n-2}} g$ satisfies

$$
\begin{aligned}
& R_{\widetilde{g}}=u^{-\frac{n+2}{n-2}}\left(-\frac{4(n-1)}{n-2} \Delta_{g} u+R_{g} u\right)=0 \text { in } M, \\
& H_{\widetilde{g}}=u^{-\frac{n}{n-2}} B_{g} u=f u^{q-\frac{n}{n-2}} \text { on } \partial M
\end{aligned}
$$

by (5.1) and (5.20). In particular, we have $H_{\widetilde{g}}\left(x_{1}\right)=f\left(x_{1}\right) u\left(x_{1}\right)^{q-\frac{n}{n-2}}=0$, as required.

Now let $O_{G}\left(x_{1}\right)=\left\{x_{1}, \ldots, x_{k}\right\}$. Let $\left(y_{1}^{1}, \cdots, y_{n}^{1}\right)$ be normal coordinates around $x_{1} \in \partial M$, such that $v_{g}\left(x_{1}\right)=-\frac{\partial}{\partial y_{n}^{1}}$ and the second fundamental form of $\partial M$ at $x_{1}$ has a diagonal form. By the invariant under $G$, we have normal coordinates $\left(y_{1}^{i}, \cdots, y_{n}^{i}\right)$ around each $x_{i} \in \partial M$ which satisfy the properties described above. Let $\delta>0$ be chosen such that $B\left(x_{i}, \delta\right) \cap$ $B\left(x_{j}, \delta\right)=\varnothing$ if $i \neq j$. Let $s_{i}(x)=\max \left\{\left|y^{i}\right|, \mid y_{n}^{i}\right\}$, where $\left|y^{i}\right|^{2}=\left(y_{1}^{i}\right)^{2}+\cdots+\left(y_{n-1}^{i}\right)^{2}$, and $\psi(x)$ be a piecewise smooth function which satisfies $\psi(s)=1$ for $|s| \leq \delta, \psi(s)=0$ for $|s| \geq 2 \delta$, and $\left|\psi^{\prime}(s)\right| \leq \delta^{-1}$ for $\delta \leq|s| \leq 2 \delta$. For $i=1, \ldots, k$, we define

$$
u_{i, \epsilon}(x)=v_{i}\left(\psi \circ s_{i}\right)
$$

where

$$
v_{i}=\left(\frac{\epsilon}{\left(\epsilon+y_{i}^{n}\right)^{2}+\left|y^{i}\right|^{2}}\right)^{\frac{n-2}{2}}
$$

and let $u_{\epsilon}=\sum_{i=1}^{k} u_{i, \epsilon}$. The function $\left.u_{\epsilon}\right|_{\partial M}$ is therefore $G$-invariant and since $Q_{\frac{2(n-1)}{n-2}}\left(u_{\epsilon}\right)=$ $k^{\frac{1}{n-1}} Q_{\frac{2(n-1)}{n-2}}\left(u_{1, \epsilon}\right)$, we can deduce that (c.f. [8])

$$
\lim _{\epsilon \rightarrow 0} Q_{\frac{2(n-1)}{n-2}}\left(u_{\epsilon}\right) \leq k^{\frac{1}{n-1}} Q\left(D^{n}, \partial D^{n}, g_{D^{n}}\right),
$$

which implies (1.9) since $k=\operatorname{card} O_{G}\left(x_{1}\right)=\inf _{x \in \partial M} \operatorname{card} O_{G}(x)$.

From the proof of Theorem 1.10, we have the following:

Corollary 5.4 The $x_{i}$ in Lemma 5.3 are necessarily points of minimal orbit in $G$. And in fact they constitute exactly a minimal orbit of $G$.

Lemma 5.5 If $x$ is a point of $\partial D^{n}$, we denote by

$$
C_{x}\left(D^{n}, \partial D^{n}, g_{D^{n}}\right)=\left\{\sigma \in C\left(D^{n}, \partial D^{n}, g_{D^{n}}\right): \sigma(x)=x\right\}
$$

and

$$
I_{x}\left(D^{n}, \partial D^{n}, g_{D^{n}}\right)=\left\{\sigma \in I\left(D^{n}, \partial D^{n}, g_{D^{n}}\right): \sigma(x)=x\right\} .
$$

For any metric $g$ conformal to $g_{D^{n}}$, we can find $\tau \in C_{x}\left(D^{n}, \partial D^{n}, g_{D^{n}}\right)$ such that $\tau^{-1} I_{x}\left(D^{n}, \partial D^{n}, g\right) \tau \subset I_{x}\left(D^{n}, \partial D^{n}, g_{D^{n}}\right)$. 
Proof Note that $I_{x}\left(D^{n}, \partial D^{n}, g\right)$ is a compact subgroup of conformal transformation of $D^{n}$. Let $\psi: S_{+}^{n} \rightarrow D^{n}$ be the projection from the upper hemisphere to the unit disc. If $\varphi$ : $S_{+}^{n}-\{x\} \rightarrow \mathbb{R}_{+}^{n}$ is the stereographic projection of pole $x, \varphi \psi I_{x}\left(D_{+}^{n}, \partial D^{n}, g\right) \psi^{-1} \varphi^{-1}$ becomes a compact group of conformal transformations of $\mathbb{R}_{+}^{n}$, a subgroup of the group of isometries of a metric conformal to the Euclidean metric, and thus makes it a subcompact group of the affine group. Therefore, $\varphi \psi I_{x}\left(D^{n}, \partial D^{n}, g\right) \psi^{-1} \varphi^{-1}$ necessarily admits a fixed point. Then we can follow the argument in Lemma 2.5 to finish the proof.

\section{Locally conformally flat case for type (II)}

In this section, we prove Theorem 1.12 when $(M, \partial M, g)$ is locally conformally flat, i.e. we will show that if $(M, \partial M, g)$ is locally conformally flat, then the strict inequality holds in (1.9). Let $(M, g)$ be a compact Riemannian manifold with boundary $\partial M$, and $G$ be a subgroup of $I(M, \partial M, g)$, the group of isometries of $g$. Assume that $\partial M$ is umbilic and $M$ is not conformally diffeomorphic to $D^{n}$. We suppose that $G$ has a finite orbit, and we denote by $O_{g}\left(x_{1}\right)=\left\{x_{1}, \cdots, x_{k}\right\}$ a minimal orbit under $G$.

Suppose that $(M, \partial M, g)$ is locally conformally flat. By Lemma 3.1, we can assume that $g$ is Euclidean in the neighborhood of each the $x_{i}$. By umbilicity, we can assume that the boundary is a hyperplane. Let $\left(y_{1}^{i}, \cdots, y_{n}^{i}\right)$ be rectangluar coordinates around $x_{i} \in \partial M$ such that $\partial M$ is defined by $y_{n}^{i}=0$ locally. Let $G_{i}$ be the positive solution of $\Delta_{g} G_{i}=0$ on $M-\left\{x_{i}\right\}$ and $B_{g} G_{i}=0$ on $\partial M$. Then $G_{i}$ has the following asymptotic expansion for $r_{i}$ small:

$$
G_{i}(x)=r_{i}^{2-n}+A+\alpha(x)
$$

where $r_{i}=d\left(x_{i}, x\right), A$ is a constant, and $\alpha(x)$ is a smooth harmonic function near $x_{i}$ with $\alpha\left(x_{i}\right)=0$.

If $\sigma\left(x_{i}\right)=x_{j}$, then $G_{j} \circ \sigma=G_{i}$, and hence $\sum_{i=1}^{k} G_{i}$ is $G$-invariant. Let $\eta_{i}$ be a smooth cut-off function as in (3.4). For each $x_{i}$, we now consider (like [8]) the function $u_{\delta, \epsilon}^{i}$ defined by

$$
u_{\delta, \epsilon}^{i}= \begin{cases}v_{\epsilon}^{i}(x), & \text { for } x \in M \cap B_{\delta}\left(x_{i}\right) \\ W_{i}(x)\left(G_{i}(x)-\eta_{i}(x) \alpha_{i}(x)\right), & \text { for } x \in M \cap\left(B_{2 \delta}\left(x_{i}\right)-B_{\delta}\left(x_{i}\right)\right) \\ W_{i}(x) G_{i}(x), & \text { for } x \in M-B_{2 \delta}\left(x_{i}\right) .\end{cases}
$$

where

$$
v_{\epsilon}^{i}(x)=\left(\frac{\epsilon}{\left(\epsilon+y_{n}^{i}\right)^{2}+r_{i}^{2}}\right)^{(n-2) / 2}
$$

and

$$
W_{i}(x)=\left(\frac{\epsilon}{\epsilon^{2}+\delta^{2}+2 \epsilon y_{n}^{i} \eta_{i}\left(r_{i}\right)}\right)^{(n-2) / 2}\left(\delta^{2-n}+A\right)^{-1} .
$$

Observe that the function $u_{\delta, \epsilon}^{i}$ is continuous across $\partial B_{\delta}$ and $\partial B_{2 \delta}$. 
We set $u_{\delta, \epsilon}=\sum_{i=1}^{k} u_{\delta, \epsilon}^{i}$ so that the function $u_{\delta, \epsilon}$ is $G$-invariant. To simplify the notations, we will write $u=u_{\delta, \epsilon}, u_{i}=u_{\delta, \epsilon}^{i}$. We compute

$$
\begin{aligned}
\frac{4(n-1)}{n-2} \int_{M}\left|\nabla_{g} u\right|^{2} d V_{g}+2 \int_{\partial M} H_{g} u^{2} d A_{g} \\
=\sum_{i=1}^{k}\left(\frac{4(n-1)}{n-2} \int_{M}\left|\nabla_{g} u_{i}\right|^{2} d V_{g}+2 \int_{\partial M} H_{g} u_{i}^{2} d A_{g}\right) \\
\quad+\sum_{i \neq j}\left(\frac{4(n-1)}{n-2} \int_{M}\left\langle\nabla_{g} u_{i}, \nabla_{g} u_{j}\right\rangle d V_{g}+2 \int_{\partial M} H_{g} u_{i} u_{j} d A_{g}\right) \\
=k\left(\frac{4(n-1)}{n-2} \int_{M}\left|\nabla u_{1}\right|^{2} d V_{g}+2 \int_{\partial M} H_{g} u_{1}^{2} d A_{g}\right) \\
+k \sum_{i=2}^{k}\left(\frac{4(n-1)}{n-2} \int_{M}\left\langle\nabla_{g} u_{1}, \nabla_{g} u_{i}\right\rangle d V_{g}+2 \int_{\partial M} H_{g} u_{1} u_{i} d A_{g}\right)
\end{aligned}
$$

where the second equality follows from the invariance under $G$. By the argument in [8, Theorem 4.1], we have

$$
\begin{aligned}
\frac{4(n-1)}{n-2} & \int_{M}\left|\nabla_{g} u_{1}\right|^{2} d V_{g}+2 \int_{\partial M} H_{g} u_{1}^{2} d A_{g} \\
\leq & Q\left(D^{n}, \partial D^{n}, g_{D^{n}}\right)\left(\int_{B_{\delta}\left(x_{1}\right) \cap \partial M} u_{1}^{\frac{2(n-1)}{n-2}} d A_{g}\right)^{\frac{n-2}{n-1}} \\
& -\frac{(n-2)}{2} \frac{\sigma_{n-1}}{4^{n-1}} A \epsilon^{n-2}+c \epsilon^{n-2} \delta+c \epsilon^{n-1} \delta^{1-n}+c \epsilon^{n} \delta^{-n} .
\end{aligned}
$$

On the other hand, we have

$$
\begin{aligned}
\frac{4(n-1)}{n-2} \int_{M}\left\langle\nabla_{g} u_{1}, \nabla_{g} u_{i}\right\rangle d V_{g}+2 \int_{\partial M} H_{g} u_{1} u_{i} d A_{g} \\
=\frac{4(n-1)}{n-2} \int_{M-B_{2 \delta}\left(x_{1}\right)-B_{2 \delta}\left(x_{i}\right)}\left\langle\nabla_{g} u_{1}, \nabla_{g} u_{i}\right\rangle d V_{g} \\
\quad+2 \int_{\partial M-B_{2 \delta}\left(x_{1}\right)-B_{2 \delta}\left(x_{i}\right)} H_{g} u_{1} u_{i} d A_{g} \\
\quad+\frac{4(n-1)}{n-2}\left(\int_{B_{2 \delta}\left(x_{1}\right)}\left\langle\nabla_{g} u_{1}, \nabla_{g} u_{i}\right\rangle d V_{g}+\int_{B_{2 \delta}\left(x_{i}\right)}\left\langle\nabla_{g} u_{1}, \nabla_{g} u_{i}\right\rangle d V_{g}\right)
\end{aligned}
$$

where we have used the fact that $H_{g}=0$ on $\partial M \cap B_{2 \delta}\left(x_{i}\right)$. Since $\Delta_{g} G_{i}=0$ in $M-\left\{x_{i}\right\}$ and $B_{g} G_{i}=0$ on $\partial M-\left\{x_{i}\right\}$, we find 


$$
\begin{aligned}
\frac{4(n-1)}{n-2} & \int_{M-B_{2 \delta}\left(x_{1}\right)-B_{2 \delta}\left(x_{i}\right)}\left\langle\nabla_{g} u_{1}, \nabla_{g} u_{i}\right\rangle d V_{g}+2 \int_{\partial M-B_{2 \delta}\left(x_{1}\right)-B_{2 \delta}\left(x_{i}\right)} H_{g} u_{1} u_{i} d A_{g} \\
= & \epsilon_{0}^{2} \int_{M \cap \partial B_{2 \delta}\left(x_{i}\right)} \frac{\partial G_{1}}{\partial v} G_{i} d A_{g}+\epsilon_{0}^{2} \int_{M \cap \partial B_{2 \delta}\left(x_{1}\right)} \frac{\partial G_{1}}{\partial v} G_{i} d A_{g} \\
& +\epsilon_{0}^{2} \int_{\partial M-B_{2 \delta}\left(x_{i}\right)-B_{2 \delta}\left(x_{i}\right)} \frac{\partial G_{1}}{\partial v_{g}} G_{i} d A_{g} \\
= & \epsilon_{0}^{2} \int_{M \cap \partial B_{2 \delta}\left(x_{i}\right)} \frac{\partial G_{1}}{\partial v} G_{i} d A_{g}+\epsilon_{0}^{2} \int_{M \cap \partial B_{2 \delta}\left(x_{1}\right)} \frac{\partial G_{1}}{\partial v} G_{i} d A_{g}
\end{aligned}
$$

where $v$ represents the unit normal vector to $M \cap \partial B_{2 \delta}\left(x_{1}\right)$ or $M \cap B_{2 \delta}\left(x_{i}\right)$. On $\partial B_{2 \delta}\left(x_{i}\right)$, we have $\left|\frac{\partial G_{1}}{\partial v}\right| \leq C$ and $G_{i} \leq C \delta^{2-n}$, which gives

$$
\epsilon_{0}^{2}\left|\int_{M \cap \partial B_{2 \delta}\left(x_{i}\right)} \frac{\partial G_{1}}{\partial v} G_{i} d A_{g}\right| \leq C \delta \epsilon_{0}^{2}
$$

On the other hand, on $\partial B_{2 \delta}\left(x_{1}\right)$, we have $\frac{\partial G_{1}}{\partial v}=r_{1}^{1-n}+O(1)$ and $G_{i}=G_{i}\left(x_{1}\right)+O\left(r_{1}\right)$. We thus obtain

$$
\epsilon_{0}^{2}\left|\int_{M \cap \partial B_{2 \delta}\left(x_{1}\right)} \frac{\partial G_{1}}{\partial v} G_{i} d A_{g}\right|=\epsilon_{0}^{2}\left|G_{i}\left(x_{1}\right)\right|+O\left(\epsilon_{0}^{2} \delta\right) .
$$

Moreover, for $i \neq j$, since $\Delta_{g} u_{j}=0$ in $B_{2 \delta}\left(x_{i}\right)$, we find

$$
\begin{aligned}
\int_{B_{2 \delta}\left(x_{i}\right)}\left\langle\nabla_{g} u_{i}, \nabla_{g} u_{j}\right\rangle d V_{g} & =\int_{M \cap \partial B_{2 \delta}\left(x_{i}\right)} u_{i} \frac{\partial u_{j}}{\partial v} d A_{g}+\int_{\partial M \cap B_{2 \delta}\left(x_{i}\right)} u_{i} \frac{\partial u_{j}}{\partial v_{g}} d A_{g} \\
& =\int_{M \cap \partial B_{2 \delta}\left(x_{i}\right)} u_{i} \frac{\partial u_{j}}{\partial v} d A_{g}
\end{aligned}
$$

and with the definition of $u_{i}$, we see that

$$
\left|\frac{\partial u_{j}}{\partial v}\right| \leq C \epsilon_{0} \text { and }\left|u_{i}\right| \leq C \epsilon_{0} \delta^{2-n} \quad \text { on } \partial B_{2 \delta}\left(x_{i}\right) .
$$

Hence, we obtain

$$
\left|\int_{B_{2 \delta}\left(x_{i}\right)}\left\langle\nabla_{g} u_{i}, \nabla_{g} u_{j}\right\rangle d V_{g}\right| \leq C \delta \epsilon_{0}^{2} .
$$

Combining all these estimates yields

$$
\begin{aligned}
& \frac{4(n-1)}{n-2} \int_{M}\left|\nabla_{g} u\right|^{2} d V_{g}+2 \int_{\partial M} H_{g} u^{2} d A_{g} \\
& \leq k Q\left(D^{n}, \partial D^{n}, g_{D^{n}}\right)\left(\int_{B_{\delta}\left(x_{1}\right) \cap \partial M} u_{1}^{\frac{2(n-1)}{n-2}} d A_{g}\right)^{\frac{n-2}{n-1}} \\
& \quad-\frac{(n-2)}{2} \frac{\sigma_{n-1}}{4^{n-1}} A \epsilon^{n-2}+c \epsilon^{n-2} \delta+c \epsilon^{n-1} \delta^{1-n}+c \epsilon^{n} \delta^{-n}+k \epsilon_{0}^{2}\left(\sum_{i=2}^{k} G_{i}\left(x_{1}\right)\right) .
\end{aligned}
$$


It now remains to evaluate $\|u\|_{L^{\frac{2(n-1)}{n-2}}(\partial M, g)}^{2}$. To this end, we compute

$$
\begin{aligned}
& \int_{\partial M} u^{\frac{2(n-1)}{n-2}} d A_{g} \\
& \leq \sum_{i=1}^{k} \int_{\partial M \cap B_{\delta}\left(x_{i}\right)}\left(u_{i}+\sum_{j \neq i} u_{j}\right)^{\frac{2(n-1)}{n-2}} d A_{g} \\
& \leq \sum_{i=1}^{k} \int_{\partial M \cap B_{\delta}\left(x_{i}\right)} u_{i}^{\frac{2(n-1)}{n-2}} d A_{g}+\frac{2(n-1)}{n-2} \sum_{i=1}^{k} \sum_{j \neq i} \int_{\partial M \cap B_{\delta}\left(x_{i}\right)} u_{i}^{\frac{n}{n-2}} u_{j} d A_{g} \\
& \leq k \int_{\partial M \cap B_{\delta}\left(x_{1}\right)} u_{1}^{\frac{2(n-1)}{n-2}} d A_{g}+\frac{2(n-1) k}{n-2} \sum_{i=2}^{k} \int_{\partial M \cap B_{\delta}\left(x_{1}\right)} u_{1}^{\frac{n}{n-2}} u_{i} d A_{g}
\end{aligned}
$$

where the second inequality follows from $(a+b)^{N} \geq a^{N}+N a^{n-1} b$, and the third inequality follows from the invariant under $G$. From the definition of $u_{1}$, we have

$$
\begin{aligned}
\left\|u_{1}\right\|_{L^{\frac{2(n-1)}{n-2}}(\partial M, g)}^{\frac{2(n-2}{n-2}} & =\int_{\partial M \cap B_{\delta}\left(x_{1}\right)} u_{1}^{\frac{2(n-1)}{n-2}} d A_{g}+\int_{\partial M-B_{\delta}\left(x_{1}\right)} u_{1}^{\frac{2(n-1)}{n-2}} d A_{g} \\
& =\int_{\partial M \cap B_{\delta}\left(x_{1}\right)} u^{\frac{2(n-1)}{n-2}} d A_{g}+\int_{\partial M-B_{\delta}\left(x_{1}\right)} W_{1}^{\frac{2(n-1)}{n-2}}\left(G_{1}-\eta_{1} \alpha_{1}\right)^{\frac{2(n-1)}{n-2}} d A_{g}
\end{aligned}
$$

and

$$
\begin{aligned}
\int_{\partial M \cap B_{\delta}\left(x_{1}\right)} u_{1}^{\frac{2(n-1)}{n-2}} d A_{g} & =\sigma_{n-2} \int_{0}^{\delta / \epsilon}\left(1+t^{2}\right)^{-(n-1)} t^{n-2} d t \\
& =\sigma_{n-2} \int_{0}^{\infty}\left(1+t^{2}\right)^{-(n-1)} t^{n-2} d t-\sigma_{n-2} \int_{\delta / \epsilon}^{\infty}\left(1+t^{2}\right)^{-(n-1)} t^{n-2} d t \\
& =2^{-(n-1)} \sigma_{n-1}+o\left(\epsilon_{0}^{2}\right) .
\end{aligned}
$$

Likewise,

$$
\begin{aligned}
\int_{\partial M \cap B_{\delta}\left(x_{1}\right)} u_{1}^{\frac{n}{n-2}} u_{i} d A_{g}= & \int_{\partial M \cap B_{\delta}\left(x_{1}\right)} u_{1}^{\frac{n}{n-2}} W_{i} G_{i} d A_{g} \\
= & \epsilon_{0} \int_{\partial M \cap B_{\delta}\left(x_{1}\right)} u_{1}^{\frac{n}{n-2}} G_{i} d A_{g} \\
= & \epsilon_{0} G_{i}\left(x_{1}\right) \int_{\partial M \cap B_{\delta}\left(x_{1}\right)} u_{1}^{\frac{n}{n-2}} d A_{g}+\epsilon_{0} \int_{\partial M \cap B_{\delta}\left(x_{1}\right)} u_{1}^{\frac{n}{n-2}} O\left(r_{1}\right) d A_{g} \\
= & \epsilon_{0} G_{i}\left(x_{1}\right) \epsilon^{\frac{n-3}{2}} \sigma_{n-2} \int_{0}^{\delta / \epsilon}\left(1+t^{2}\right)^{-\frac{n+1}{2}} t^{n-2} d t \\
& +\epsilon_{0} \int_{\partial M \cap B_{\delta}\left(x_{1}\right)} u_{1}^{\frac{n}{n-2}} O\left(r_{1}\right) d A_{g} .
\end{aligned}
$$

where the second equality follows from

$$
W_{i}(x)=\left(\frac{\epsilon}{\epsilon^{2}+\delta^{2}}\right)^{\frac{n-2}{2}}\left(\delta^{2-n}+A\right)^{-1}=: \epsilon_{0} \text { for } x \in \partial M \cap B_{\delta}\left(x_{1}\right)
$$


by the definition of $W_{i}$ in (6.1). Therefore, as in Sect. 3, we obtain

$$
\begin{gathered}
Q_{\frac{2(n-1)}{n-2}}\left(u_{\delta, \epsilon}\right) \leq k^{\frac{1}{n-1}} Q\left(D^{n}, \partial D^{n}, g_{D^{n}}\right)-\epsilon_{0}^{2}\left(C_{0} A+C_{2}(k-1)\right) \\
+O\left(\epsilon_{0}^{2} \delta\right)+o\left(\epsilon_{0}^{2}\right)
\end{gathered}
$$

where $C_{0}$ and $C_{2}$ are strictly positive constants. In particular, we can find $\delta$ and $\epsilon$ sufficiently small so that $Q_{\frac{2(n-1)}{n-2}}\left(u_{\delta, \epsilon}\right)<k^{\frac{1}{n-1}} Q\left(D_{+}^{n}, \partial D_{+}^{n}, g_{D_{+}^{n}}\right)$ if $C_{0} A+C_{2}(k-1)>0$. The positive mass theorem guarantees that $A>0$ (cf. [9, Appendix]. Therefore we can conclude that $\mu_{\frac{2(n-1)}{n-2}}(G)<k^{\frac{1}{n-1}} Q\left(D_{+}^{n}, \partial D_{+}^{n}, g_{D_{+}^{n}}\right)$.

Now we suppose $M=D^{n}$. Then, since $M$ is locally conformally flat, we can follow the above argument and eventually obtain (6.2). But, in this case, the constant $A$ is zero. If $k \geq 2$, we still have $C_{0} A+(k-1) C_{2}=(k-1) C_{2}>0$.

Therefore, we suppose $k=$ card $O_{G}\left(x_{1}\right)=1$, i.e. $G$ admits a fixed point (denote by $x$ ). Since $x$ is a fixed point, $G$ becomes a subgroup of $I_{x}\left(D^{n}, \partial D^{n}, g\right)$. Then, by Lemma 5.5, there exist $\tau \in C_{x}\left(D^{n}, \partial D^{n}, g_{D^{n}}\right)$ so that $G \subset \tau^{-1} I_{x}\left(D^{n}, \partial D^{n}, g_{D^{n}}\right) \tau$. Let $f, \varphi>0$ be smooth functions such that

$$
\left(\tau^{-1}\right)^{*} g_{D^{n}}=f^{\frac{4}{n-2}} g_{D^{n}}, \quad g=\varphi^{\frac{4}{n-2}} g_{D^{n}} .
$$

We set $u(x)=1 / \varphi(x) f(\tau(x))$. First, we show that $u$ is $G$-invariant. To see this, consider $\sigma \in G$ and $i \in I_{x_{1}}\left(D^{n}, \partial D^{n}, g_{D^{n}}\right)$ which satisfies $\sigma=\tau^{-1} i \tau$. Then

$$
\begin{aligned}
\sigma^{*} g & =\tau^{*} i^{*}\left(\tau^{-1}\right)^{*} g \\
& =\left(\tau^{*} i^{*}\right)\left(\left(\varphi \circ \tau^{-1}\right)^{\frac{4}{n-2}} f^{\frac{4}{n-2}} g_{S_{+}^{n}}\right) \\
& =((\varphi \circ \sigma)(f \circ i \circ \tau))^{\frac{4}{n-2}}(f \circ \tau)^{-\frac{4}{n-2}} g_{S_{+}^{n}} .
\end{aligned}
$$

But since $\sigma^{*} g=g$, we get $(\varphi \circ \tau)((f \circ \tau) \circ \sigma)=\varphi(f \circ \tau)$. This shows that the invariance of $u$ under $\sigma$, hence under $G$. Since $\varphi u=1 /(f \circ \tau)$ and $\tau^{*} g_{D^{n}}=(f \circ \tau)^{-4 /(n-2)} g_{D^{n}}$, we can easily verify that $Q_{\frac{2(n-1)}{n-2}}(u)=Q\left(D^{n}, \partial D^{n}, g_{D^{n}}\right)$. This shows that $\mu_{\frac{2(n-1)}{n-2}}(G)=$ $Q\left(D^{n}, \partial D^{n}, g_{D^{n}}\right)$.

\section{The case of dimension 3, 4 and 5 for type (II)}

In this section, we prove Theorem 1.12 when $3 \leq n \leq 5$. Basically, it can be proved by the same argument as Type I. Therefore, we will only consider the case when $n=5$.

Suppose that $n=5$. Let $\left(y_{1}^{i}, \cdots, y_{n}^{i}\right)$ denote a normal rectangular coordinate system centered at $x_{i}$. Let $r_{i}=\left|y^{i}\right|, \zeta=y_{i} /\left|y^{i}\right|$. Then the metric $g$ of $M$ can be locally written as

$$
g=d r_{i}^{2}+r_{i}^{2} h_{r_{i}}
$$

where $h_{r_{i}}$ is a metric on $S_{+}^{n-1}$ with $h_{0}$ being the standard metric. Given $\rho>0$ with $\rho$ small, let $\xi\left(r_{i}\right)$ be a smooth nonincreasing function satisfying $\xi\left(r_{i}\right)=1$ for $r_{i} \leq \rho$, and $\xi\left(r_{i}\right)=0$ for $r_{i} \geq 2 \rho$; let $\left|\xi^{\prime}\left(r_{i}\right)\right| \leq c \rho^{-1}$ and let $\left|\xi^{\prime \prime}\left(r_{i}\right)\right| \leq c \rho^{-2}$ for all $r_{i}>0$. We define the metric ${ }^{\rho} g$ on $M$ by setting ${ }^{\rho} g=0$ on $M-B_{2 \rho}^{+}$and

$$
{ }^{\rho} g=d r_{i}^{2}+r_{i}^{2}\left(\xi\left(r_{i}\right) h_{0}+\left(1-\xi\left(r_{i}\right)\right) h_{r_{i}}\right), \quad r_{i} \leq 2 \rho .
$$


Thus ${ }^{\rho} g$ is Euclidean in $B_{\rho}^{+}$and coincides with $g$ outside $B_{2 \rho}^{+}$. It is easy to check that the curvature tensor of ${ }^{\rho} \mathrm{g}$ is bounded, independent of $\rho$. It follows from [8, Theorem 7.1] that the boundary $\partial M$ is totally geodesic with respect to the metric ${ }^{\rho} g$.

Let $L_{\rho}$ and $B_{\rho}$ be the following linear operator taken in terms of ${ }^{\rho} g$ :

$$
L_{\rho}=\Delta_{\rho}, \quad B_{\rho}=\frac{2(n-1)}{n-2} \frac{\partial}{\partial v_{\rho}}+H_{\rho} .
$$

Let $\lambda_{\rho}$ denote the lowest eigenvalue of $L_{\rho}$, and $\lambda$ the lowest eigenvalue of $L$. Then since $g$ is conformally related to a metric with positive scalar curvature, where the boundary is minimal, we have $\lambda>0$. Let $G_{i}$ be the multiple of the Green's function of $(L, B)$ with pole at $x_{i}$ and normalize so that $\lim _{\left|y^{i}\right| \rightarrow 0}\left|y^{i}\right|^{3} G_{i}\left(y^{i}\right)=1$. Note that ${ }^{\rho} g$ converges in the $C^{1}$-norm to $g$ as $\rho \searrow 0$ and $R_{\rho}$ is uniformly bounded. Thus, for $\rho$ sufficiently small, $\lambda_{\rho}>0$. Hence we can consider $G_{i, \rho}$ to be the positive Green's function $\left(L_{\rho}, B_{\rho}\right)$. Normalize the function $G_{i, \rho}$ such that $\lim _{\left|y^{i}\right| \rightarrow 0}\left|y^{i}\right|^{3} G_{i, \rho}=1$. Consider $\tilde{M}=M \cup \partial M \cup M$ as the double of $M$ with the standard metric induced from $M$. Then we can show that the function $G_{i, \rho}$ converges to $G_{i}$ as $\rho \rightarrow 0$ in the $C^{2}$-norm on compact subsets of $\bar{M}-\left\{x_{i}\right\}$ by applying Lemma 1 in [17]. Since the metric ${ }^{\rho} g$ are Euclidean in $B_{\rho}\left(x_{i}\right)$, the function $G_{i, \rho}$ is harmonic in $B_{\rho}$ and hance has an expansion for $\left|y^{i}\right|$ small,

$$
G_{i, \rho}\left(y^{i}\right)=\left|y^{i}\right|^{-3}+A_{\rho}+O\left(\left|y^{i}\right|\right), \quad A_{\rho} \geq 0 .
$$

We define $u_{\delta, \epsilon}^{i}$, and $u_{\delta, \epsilon}$ as in the previous section. Then it follows from (7.16) in [8] that

$$
\begin{aligned}
\frac{16}{3} \int_{M}\left|\nabla_{g} u_{1}\right|^{2} d & V_{g}+2 \int_{\partial M} H_{g} u_{1}^{2} d A_{g} \\
\leq & Q\left(D^{5}, \partial D^{5}, g_{D^{5}}\right)\left(\int_{B_{\rho_{0}} \cap \partial M} u_{1}^{\frac{8}{3}} d A_{g}\right)^{\frac{3}{4}} \\
& -\frac{3}{2} \frac{\sigma_{4}}{4^{4}} A_{\rho} \epsilon^{3}+c \epsilon^{3} \rho_{0}+c \epsilon^{5} \rho_{0}^{-5}+c \epsilon^{4} \rho_{0}^{-4}+c_{1} \rho \epsilon^{3},
\end{aligned}
$$

where $c$ depends on $\rho$ but $c_{1}$ does not. Then we can show that

$$
Q_{\frac{8}{3}}\left(u_{\delta, \epsilon}\right) \leq k^{\frac{1}{4}} Q\left(D^{5}, \partial D^{5}, g_{D^{5}}\right)-\epsilon_{0}^{2}\left(C_{0} A+C_{2}(k-1)\right)+\text { negligible terms }
$$

where $C_{0}$ and $C_{2}$ are positive constants. Since $M$ is not conformally equivalent to $S_{+}^{5}$, if follows from [8, Section7] that

$$
\liminf _{\rho \rightarrow 0} A_{\rho}>0 .
$$

Fixing $\rho$ small, then fixing $\rho_{0}$, and finally choosing $\epsilon_{0}$ sufficiently small, we thus have $\mu_{\frac{8}{3}}(G)<k^{1 / 4} Y\left(D^{5}, \partial D^{5}, g_{D^{5}}\right)$.

\section{Appendix}

In this Appendix, we will give the proof of Lemma 2.3. To this end, we follow the argument in [18, Theorem3.1]. First, we have the following: 
Lemma 8.1 Let $u \in C^{\infty}(M, g)$ be a solution of the equation

$$
\left\{\begin{array}{r}
\frac{4(n-1)}{n-2} \Delta_{g} u+R_{g} u=P u \text { in } M ; \\
\frac{\partial u}{\partial v_{g}}=0 \text { on } \partial M .
\end{array}\right.
$$

(i) For any $\sigma<Y\left(S_{+}^{n}, \partial S_{+}^{n}, g_{S_{+}^{n}}\right)$, there exist constants $q_{0}>\frac{2 n}{n-2}, r_{0}>0$ such that, whenever for some $r<r_{0}$ and some $x_{0} \in \bar{M}$ there holds $\|P\|_{L^{n / 2}\left(B_{2 r}\left(x_{0}\right)\right)} \leq \sigma$, then

$$
\|u\|_{L^{q_{0}}\left(B_{r}\left(x_{0}\right)\right)}<C\|u\|_{L^{\frac{2 n}{n-2}}\left(B_{2 r}\left(x_{0}\right)\right)} .
$$

(ii) For any $q>\frac{2 n}{n-2}$, any $r>0$, there exist a constant $C=C(q, r)>0$ such that

$$
\begin{gathered}
\|u\|_{L^{q}\left(B_{3 r}\left(x_{0}\right)\right)} \leq C\|u\|_{L^{\frac{2 n}{n-2}\left(B_{2 r}\left(x_{0}\right)\right)}}, \\
\text { whenever there holds }\|P\|_{L^{n / 2}\left(B_{4 r}\left(x_{0}\right)\right)}<2 n Y\left(S_{+}^{n}, \partial S_{+}^{n}, g_{S_{+}^{n}}\right) /((n-2) q) .
\end{gathered}
$$

Proof (i) This assertion will follow by performing one step of a Moser iteration. Choose some smooth cut-off function $\eta \in C_{0}^{1}\left(B_{2 r}\left(x_{0}\right)\right)$ satisfying $\eta(x)=1$ in $B_{r}\left(x_{0}\right)$ and $\left|\nabla_{g} \eta\right|<C / r$. For suitable $p \geq 1$, we then let $v=u^{2 p-1} \eta^{2}$. Multiplying the first equation of (8.1) by $v$, using integrating by parts and the second equation of (8.1), we have

$$
\int_{M}\left(\frac{4(n-1)}{n-2}\left\langle\nabla_{g} u, \nabla_{g} v\right\rangle+R_{g} u v\right) d V_{g}=\int_{M} P u v d V_{g} .
$$

Let $w=u^{p} \eta$. Then $|w|^{2}=u v$, and Young's inequality implies that

$$
\left|\nabla_{g} w\right|^{2} \leq \frac{p+1}{2}\left(\left\langle\nabla_{g} u, \nabla_{g} v\right\rangle+u^{2 p}\left|\nabla_{g} \eta\right|^{2}\right) .
$$

It follows from (8.2), (8.3), and the Hölder's inequality that

$$
\begin{aligned}
& \frac{2}{p+1}\left(\int_{M} \frac{4(n-1)}{n-2}\left|\nabla_{g} w\right|^{2}+R_{g} w^{2} d V_{g}\right)-\frac{4(n-1)}{n-2} \int_{M} u^{2 p}\left|\nabla_{g} \eta\right|^{2} d V_{g} \\
& \leq \int_{M}\left|P\left\|\left.w\right|^{2} d V_{g} \leq\right\| P\left\|_{L^{\frac{n}{2}}\left(B_{2 r}\left(x_{0}\right) \cap M\right)}\right\| w \|_{L^{2}}^{2 \frac{2 n}{n-2}\left(B_{2 r}\left(x_{0}\right) \cap M\right)} \cdot\right.
\end{aligned}
$$

On the other hand, for sufficiently small $r_{0}>0$ the metric $g$ on $B_{r_{0}}\left(x_{0}\right)$ in normal coordinates around $x_{0}$ differs arbitrarily little from the Euclidean metric. Hence for function $w$ with support in $B_{2 r}\left(x_{0}\right)$ the following Sobolev-type inequality holds with constant $A$ arbitrarily close to $Y\left(S_{+}^{n}, \partial S_{+}^{n}, g_{S_{+}^{n}}\right)$ :

$$
\|w\|_{L^{\frac{2 n}{n-2}(M, g)}}^{2} \leq A^{-1} \int_{M}\left(\frac{4(n-1)}{n-2}|\nabla w|_{g}^{2}+R_{g} w^{2}\right) d V_{g} .
$$

Let $\sigma=a Y\left(S_{+}^{n}, \partial S_{+}^{n}, g_{S_{+}^{n}}\right)$, where $a<1$. Then by (8.4), (8.5), and the assumption $\|P\|_{L^{n / 2}\left(B_{2 r}\left(x_{0}\right) \cap M\right)} \leq \sigma$, we have

$$
\int_{M} \frac{4(n-1)}{n-2}\left|\nabla_{g} w\right|^{2}+R_{g} w^{2} d V_{g} \leq\left(\frac{2}{p+1}-\sigma A^{-1}\right)^{-1} \frac{4(n-1)}{n-2} \int_{M} u^{2 p}\left|\nabla_{g} \eta\right|^{2} d V_{g} .
$$


For $1<p<\min \left\{\frac{3-a}{1+a}, \frac{n}{n-2}\right\}$ and $r<r_{0}=r_{0}(a)$, set $C^{*}=\frac{1-a}{4} \cdot \frac{n-2}{4(n-1)} Y\left(S_{+}^{n}, \partial S_{+}^{n}, g_{S_{+}^{n}}\right)$. Using (8.4), (8.5), our smallness assumption and the Hölder's inequality we then obtain

$$
\begin{aligned}
& C^{*}\|u\|_{L^{\frac{2 n p}{n-2}\left(B_{r}\left(x_{0}\right) \cap M\right)}}^{2 p}=C^{*}\|w\|_{L^{\frac{2 n}{n-2}}\left(B_{r}\left(x_{0}\right) \cap M\right)}^{2} \\
& \leq C^{*}\|w\|_{L^{\frac{2 n}{n-2}}(M)}^{2} \\
& \leq C^{*} A^{-1} \int_{M}\left(\frac{4(n-1)}{n-2}\left|\nabla_{g} w\right|^{2}+R_{g} w^{2}\right) d V_{g} \\
& \leq \int_{M} u^{2 p}\left|\nabla_{g} \eta\right|^{2} d V_{g} \\
& \leq C r^{-2}\|u\|^{2 p}{ }^{\frac{2 n}{n-2}\left(B_{2 r}\left(x_{0}\right) \cap M\right)} .
\end{aligned}
$$

By setting $q_{0}=\frac{2 p n}{n-1}>\frac{2 n}{n-1}$, we thus complete the proof of the first claim.

(ii) We perform finitely many steps of a Moser iteration. For $1<p \leq \frac{(n-2) q}{2 n}$ and $\eta \in C^{1}\left(B_{4 r}\left(x_{0}\right)\right)$, we define the test function $v=u^{2 p-1} \eta^{2}$, leading to (8.6) for $w=u^{p} \eta$ as above. From (8.5) and our smallness assumption we derive

$$
\frac{n-2}{4(n-1)} Y\left(S_{+}^{n}, \partial S_{+}^{n}, g_{S_{+}^{n}}\right)\left(\frac{2}{1+p}-\frac{(n-2) q}{2 n}\right)\|w\|_{L^{\frac{2 n}{n-2}}}^{2} \leq \int_{M} u^{2 p}\left|\nabla_{g} \eta\right|^{2} d V_{g}
$$

Choose $p_{1} \in\left(1, \frac{n}{n-2}\right]$ maximal such that $q=2 p_{1}\left(\frac{n}{n-2}\right)^{m}$ for some $m \in \mathbb{N}$. For $i=$ $1, \cdots, m+1$ then let $p_{i}=p_{1}\left(\frac{n}{n-2}\right)^{i-1}, r_{i}=r\left(3+2^{1-i}\right), \eta=\eta_{i}$ such that $\eta \equiv 1$ in $B_{r_{i+1}}\left(x_{0}\right), \eta \equiv 0$ in $M \backslash B_{r_{i}}\left(x_{0}\right)$, and $\left|\nabla_{g} \eta\right|<C 2^{i} / r$. From (8.7), for $1 \leq i \leq m$ we then obtain

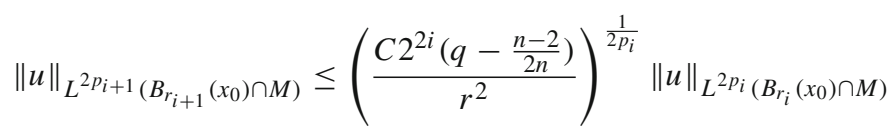

which can be iterated to obtain the result.

Lemma 8.2 Let $g_{k}=u_{k}^{\frac{4}{n-2}} g$, where $0<u_{k} \in C^{\infty}\left(M, g_{0}\right), k \in \mathbb{N}$, be a family of conformal metrics with unit volume $\int_{M} u_{k}^{\frac{2 n}{n-2}} d V_{g}=1$ and satisfying

$$
\int_{M} R_{g_{k}} d V_{g_{k}} \leq C_{0}, \quad \int_{M}\left|R_{g_{k}}-\bar{R}_{g_{k}}\right|^{p} d V_{g_{k}} \leq C_{0}
$$

for all $k$ and some $p>n / 2$ where $\bar{R}_{g_{k}}=\int_{M} R_{g_{k}} d V_{g_{k}}$. Then, either $(i)$ the sequence $\left(u_{k}\right)$ is uniformly bounded in $W^{2, p}(M, g) \hookrightarrow L^{\infty}(M, g)$, or (ii) there exists a subsequence $\left(u_{k}\right)$ and finitely many points $x_{i}, \cdots, x_{L} \in \bar{M}$ such that for any $r>0$ and any $l \in\{1, \cdots, €\}$ there holds

$$
\liminf _{k \rightarrow \infty}\left(\int_{B_{r}\left(x_{l}\right) \cap M}\left|R_{g_{k}}\right|^{n / 2} d V_{g_{k}}\right)^{2 / n} \geq Y\left(S_{+}^{n}, \partial S_{+}^{n}, g_{S_{+}^{n}}\right) .
$$

Moreover, the sequence $\left(u_{k}\right)$ is bounded in $W^{2, p}$ on any compact subset of $\bar{M} \backslash\left\{x_{1}, \cdots, x_{L}\right\}$. 
Proof Fix a point $x_{0} \in \bar{M}$ and assume that for some $r>0$ there holds

$$
\sup _{k}\left(\int_{B_{2 r}\left(x_{0}\right) \cap M}\left|R_{g_{k}}\right|^{\frac{n}{2}} d V_{g_{k}}\right)^{\frac{2}{n}} \leq \sigma<Y\left(S_{+}^{n}, \partial S_{+}^{n}, g_{S_{+}^{n}}\right) .
$$

Writing the Yamabe equation for $u_{k}$ as

$$
-\frac{4(n-1)}{n-2} \Delta_{g} u_{k}+R_{g} u_{k}=R_{g_{k}} u_{k}^{\frac{n+2}{n-2}}=P_{k} u_{k},
$$

we have $P_{k}=R_{g_{k}} u_{k}^{\frac{4}{n-2}} \in L^{n / 2}\left(B_{2 r}\left(x_{0}\right) \cap M\right)$ with

$$
\left\|P_{k}\right\|_{L^{\frac{n}{2}}\left(B_{2 r}\left(x_{0}\right) \cap M\right)}=\left(\int_{B_{2 r}\left(x_{0}\right) \cap M}\left|R_{g_{k}}\right|^{\frac{n}{2}} d V_{g_{k}}\right)^{\frac{2}{n}} \leq \sigma<Y\left(S_{+}^{n}, \partial S_{+}^{n}, g_{S_{+}^{n}} .\right.
$$

Clearly, we may assume that $r \leq r_{0}(\sigma)$ as determined in Lemma 8.1. Lemma 8.1 and the assumption $\operatorname{Vol}\left(M, g_{k}\right)=1$ then imply that $\left(u_{k}\right)$ is bounded in $L^{q_{0}}\left(B_{r}\left(x_{0}\right) \cap M\right)$ for some $q_{0}>\frac{2 n}{n-2}$. In particular,

$$
\operatorname{Vol}\left(B_{r}\left(x_{0}\right), g_{k}\right) \rightarrow 0 \text { as } r \rightarrow 0
$$

uniformly in $k \in \mathbb{N}$.

Choose $q>\frac{n}{2}$ such that

$$
\frac{1}{p_{1}}:=\max \left\{\frac{1}{p}+\frac{(p-2) n+2 p}{p q(n-2)}, \frac{n+2}{q(n-2)}\right\}<\frac{2}{n} .
$$

By Hölder's inequality, for any $r>0$ we have

$$
\begin{aligned}
& \left(\int_{B_{r}\left(x_{l}\right) \cap M}\left|R_{g_{k}}\right| d V_{g_{k}}\right)^{\frac{2}{n}} \leq \bar{R}_{g_{k}}\left(\int_{B_{r}\left(x_{l}\right) \cap M} d V_{g_{k}}\right)^{\frac{2}{n}}+\left(\int_{B_{r}\left(x_{l}\right) \cap M}\left|R_{g_{k}}-\bar{R}_{g_{k}}\right|^{\frac{n}{2}} d V_{g_{k}}\right)^{\frac{2}{n}} \\
& \leq \bar{R}_{g_{k}}\left(\int_{B_{r}\left(x_{l}\right) \cap M} d V_{g_{k}}\right)^{\frac{2}{n}}+\left(\int_{B_{r}\left(x_{l}\right) \cap M}\left|R_{g_{k}}-\bar{R}_{g_{k}}\right|^{p} d V_{g_{k}}\right)^{\frac{1}{p}}\left(\int_{B_{r}\left(x_{l}\right) \cap M} d V_{g_{k}}\right)^{\frac{2}{n}-\frac{1}{p}} .
\end{aligned}
$$

Using (8.8), (8.11) and (8.12), and replacing $r$ by a smaller radius if necessary, we can achieve that $\left\|P_{k}\right\|_{L^{\frac{n}{2}}\left(B_{4 r}\left(x_{0}\right) \cap M\right)}<\frac{2 n}{q(n-1)} Y\left(S_{+}^{n}, \partial S_{+}^{n}, g_{S_{+}^{n}}\right)$. By Lemma 8.1 and $\operatorname{Vol}\left(M, g_{k}\right)=1$, then $\left(u_{k}\right)$ is bounded in $L^{q}\left(B_{3 r}\left(x_{0}\right) \cap M\right)$, and by choice of $q$ we obtain uniform bounds for

$$
\begin{aligned}
\frac{4(n-1)}{n-2} \Delta_{g} u_{k} & =R_{g_{k}} u^{\frac{n+2}{n-2}}-R_{g} u_{k} \\
& =\left(R_{g_{k}}-\bar{R}_{g_{k}}\right) u_{k}^{\frac{n+2}{n-2}}+\bar{R}_{g_{k}} u_{k}^{\frac{n+2}{n-2}}-R_{g} u_{k} \in L^{p_{1}}\left(B_{3 r}\left(x_{0}\right) \cap M\right) .
\end{aligned}
$$

Then Calderón-Zygmund inequality and Sobolev's embedding now yield the estimate

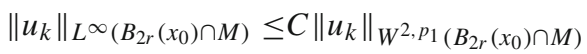

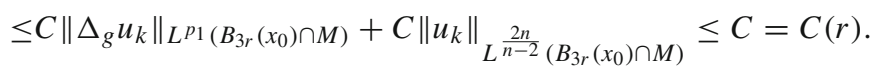

Going back to the previous equation, we then find that

$$
\left|\Delta_{g} u_{k}\right| \leq C\left|R_{g_{k}}-\bar{R}_{g_{k}}\right| u_{k}^{\frac{n+2}{n-2}}+\bar{R}_{g_{k}} u_{k}^{\frac{n+2}{n-2}}+R_{g} u_{k}
$$


is bounded in $L^{p}\left(B_{2 r}\left(x_{0}\right) \cap M\right)$. Thus, by the Calderón-Zygmund inequality, $u_{k} \in$ $W^{2, p}\left(B_{r}\left(x_{2}\right) \cap M\right)$ with

$$
\left\|u_{k}\right\|_{W^{2, p}\left(B_{r}\left(x_{0}\right) \cap M\right)} \leq C=C(r) .
$$

Now assume that condition (8.10) is satisfied for every $x \in \bar{M}$ and some radius $r=$ $r(x)>0$. The cover $\left(B_{r(x)}(x)\right)_{x \in \bar{M}}$ of $\bar{M}$ admits a finite subcover $B_{r_{i}}(x)$, where $r_{i}=r\left(x_{i}\right)$, $1 \leq i \leq I$. From (8.13) we then obtain the desired uniform bound

$$
\left\|u_{k}\right\|_{W^{2, p}(M)} \leq I \max _{1 \leq i \leq I} C\left(r_{i}\right) .
$$

If (8.10) does not hold for every $x$ with some $r=r(x)>0$, we iteratively determine points $x_{l}, l \in \mathbb{N}$, and a subsequence $\left(u_{k}\right)$ such that for any $r>0$ and any $l$ condition (8.9) is valid. This iteration terminates after finitely many steps. Indeed, given $x_{1}, \cdots, x_{L}$, choose $0<r<\min _{i \neq j} \operatorname{dist}\left(x_{i}, x_{j}\right) / 2$. Then (8.9) yields the bound

$$
\begin{aligned}
L \cdot Y\left(S_{+}^{n}, \partial S_{+}^{n}, g_{S_{+}^{n}}\right)^{\frac{n}{2}} & \leq \sum_{l=1}^{L} \liminf _{k \rightarrow \infty} \int_{B_{r}\left(x_{l}\right) \cap M}\left|R_{g_{k}}\right|^{\frac{n}{2}} d V_{g_{k}} \\
& =\liminf _{k \rightarrow \infty} \int_{\cup_{l=1}^{L} B_{r}\left(x_{l}\right) \cap M}\left|R_{g_{k}}\right| \frac{n}{2} d V_{g_{k}} \\
& \leq \sup _{k} \int_{M}\left|R_{g_{k}}\right|^{\frac{n}{2}} d V_{g_{k}} \\
& \leq C \sup _{k}\left(\int_{M}\left|R_{g_{k}}-\bar{R}_{g_{k}}\right|^{\frac{n}{2}} d V_{g_{k}}+\bar{R}_{g_{k}}^{\frac{n}{2}}\right)<\infty .
\end{aligned}
$$

By a covering argument as above we then obtain that $\left(u_{k}\right)$ is bounded in $W^{2, p} \hookrightarrow L^{\infty}$ on any compact subset of $\bar{M} \backslash\left\{x_{1}, \cdots, x_{L}\right\}$, as claimed.

Acknowledgements The authors would like to thank the referee for his/her comments and suggestions, which improve the presentation of this paper. The first author was supported by Basic Science Research Program through the National Research Foundation of Korea (NRF) funded by the Ministry of Education, Science and Technology (Grant No. 2020R1A6A1A03047877 and 2019R1F1A1041021), and by Korea Institute for Advanced Study (KIAS) grant funded by the Korea government (MSIP). The second author was supported by a KIAS Individual Grant (MG070701) at Korea Institute for Advanced Study.

Open Access This article is licensed under a Creative Commons Attribution 4.0 International License, which permits use, sharing, adaptation, distribution and reproduction in any medium or format, as long as you give appropriate credit to the original author(s) and the source, provide a link to the Creative Commons licence, and indicate if changes were made. The images or other third party material in this article are included in the article's Creative Commons licence, unless indicated otherwise in a credit line to the material. If material is not included in the article's Creative Commons licence and your intended use is not permitted by statutory regulation or exceeds the permitted use, you will need to obtain permission directly from the copyright holder. To view a copy of this licence, visit http://creativecommons.org/licenses/by/4.0/.

\section{References}

1. Almaraz, S.: A compactness theorem for scalar-flat metrics on manifolds with boundary. Calc. Var. Partial Differ. Equ. 41(3-4), 341-386 (2011)

2. Aubin, T.: Équations différentielles non linéaires et problème de Yamabe concernant la courbure scalaire. J. Math. Pures Appl. (9) 55, 269-296 (1976)

3. Aubin, T.: Some Nonlinear Problems in Riemannian Geometry. Springer Monographs in Mathematics, Springer, Berlin (1998) 
4. Brendle, S.: A generalization of the Yamabe flow for manifolds with boundary. Asian J. Math. 6(4), 625-644 (2002)

5. Brendle, S., Chen, S.-Y.S.: An existence theorem for the Yamabe problem on manifolds with boundary. J. Eur. Math. Soc. 16(5), 991-1016 (2014)

6. Cherrier, P.: Problèmes de Neumann non linéaires sur les variétés riemanniennes. J. Funct. Anal. 57, 154-206 (1984)

7. Disconzi, M., Khuri, M.A.: Compactness and non-compactness for the Yamabe problem on manifolds with boundary. J. Reine Angew. Math. 724, 145-201 (2017)

8. Escobar, J.F.: Conformal deformation of a Riemannian metric to a scalar flat metric with constant mean curvature on the boundary. Ann. Math. 136, 1-50 (1992)

9. Escobar, J.F.: The Yamabe problem on manifolds with boundary. J. Differ. Geom. 35, 21-84 (1992)

10. Hebey, E., Vaugon, M.: Le problème de Yamabe équivariant. Bull. Sci. Math. 117, 241-286 (1993)

11. Henry, G., Madani, F.: The equivariant second Yamabe constant. J. Geom. Anal. 28(4), 3747-3774 (2018)

12. Kuiper, N.H.: On compact conformally Euclidean spaces of dimension > 2. Ann. Math. 52, 478-490 (1950)

13. Li, Y., Zhu, M.: Sharp Sobolev trace inequalities on Riemannian manifolds with boundaries. Commun. Pure Appl. Math. 50, 449-487 (1997)

14. Madani, F.: Equivariant Yamabe problem and Hebey-Vaugon conjecture. J. Funct. Anal. 258, 241-254 (2010)

15. Madani, F.: Hebey-Vaugon conjecture II. C. R. Math. Acad. Sci. Paris 350, 849-852 (2012)

16. Mayer, M., Ndiaye, C.B.: Barycenter technique and the Riemann mapping problem of Cherrier-Escobar. J. Differ. Geom. 107(3), 519-560 (2017)

17. Schoen, R.: Conformal deformation of a Riemannian metric to constant scalar curvature. J. Differ. Geom. 20, 479-495 (1984)

18. Schwetlich, H., Struwe, M.: Convergence of the Yamabe flow for "large" energies. J. Reine Angew. Math. 562, 59-100 (2003)

19. Sung, C.: Surgery and equivariant Yamabe invariant. Differ. Geom. Appl. 24(3), 271-287 (2006)

20. Trudinger, N.S.: Remarks concerning the conformal deformation of Riemannian structures on compact manifolds. Ann. Scuola Norm. Sup. Pisa (3) 22, 265-274 (1968)

21. Xu, X., Zhang, H.: Conformal metrics on the unit ball with prescribed mean curvature. Math. Ann. 365, 497-557 (2016)

22. Yamabe, H.: On a deformation of Riemannian structures on compact manifolds. Osaka Math. J. 12, 21-37 (1960)

Publisher's Note Springer Nature remains neutral with regard to jurisdictional claims in published maps and institutional affiliations. 